\title{
ALFALFA Hi Data Stacking I. Does the Bulge Quench Ongoing Star Formation in Early-Type Galaxies?
}

\author{
Silvia Fabello ${ }^{1 \star}$, Barbara Catinella ${ }^{1}$, Riccardo Giovanelli ${ }^{2}$, Guinevere Kauffmann ${ }^{1}$, \\ Martha P. Haynes ${ }^{2}$, Timothy M. Heckman ${ }^{3}$, David Schiminovich ${ }^{4}$ \\ ${ }^{1}$ Max-Planck Institut für Astrophysik, D-85741 Garching, Germany \\ ${ }^{2}$ Center for Radiophysics and Space Research, Cornell University, Ithaca, NY 14853, USA \\ ${ }^{3}$ Department of Physics and Astronomy, The Johns Hopkins University, Baltimore, MD 21218, USA \\ ${ }^{4}$ Department of Astronomy, Columbia University, New York, NY 10027, USA
}

\begin{abstract}
We have carried out an Hi stacking analysis of a volume-limited sample of $\sim 5000$ galaxies with imaging and spectroscopic data from GALEX and the Sloan Digital Sky Survey, which lie within the current footprint of the Arecibo Legacy Fast ALFA (ALFALFA) Survey. Our galaxies are selected to have stellar masses greater than $10^{10} M_{\odot}$ and redshifts in the range $0.025<z<0.05$. We extract a sub-sample of 1833 "early-type" galaxies with inclinations less than $70^{\circ}$, with concentration indices $C>2.6$ and with light profiles that are well fit by a De Vaucouleurs model. We then stack HI line spectra extracted from the ALFALFA data cubes at the 3-D positions of the galaxies from these two samples in bins of stellar mass, stellar mass surface density, central velocity dispersion, and NUV-r colour. We use the stacked spectra to estimate the average HI gas fractions $\mathrm{M}_{\mathrm{HI}} / M_{*}$ of the galaxies in each bin. Our main result is that the Hi content of a galaxy is not influenced by its bulge. The average Hi gas fractions of galaxies in both our samples correlate most strongly with NUV-r colour and with stellar surface density. The relation between average Hi fraction and these two parameters is independent of concentration index $C$. We have tested whether the average $\mathrm{HI}$ gas content of bulge-dominated galaxies on the red sequence, differs from that of late-type galaxies on the red sequence. We find no evidence that galaxies with a significant bulge component are less efficient at turning their available gas reservoirs into stars. This result is in contradiction with the "morphological quenching" scenario proposed by Martig et al. (2009).
\end{abstract}

Key words: galaxies:evolution-galaxies: fundamental parameters-radio lines:galaxies

\section{INTRODUCTION}

Galaxies have long been known to follow well-ordered sequences in many properties (see Roberts \& Haynes 1994, for a review). In the simplest classification scheme, galaxies divide into spirals and ellipticals. In more complex schemes, these classes are partitioned into additional sub-classes. Irrespective of the exact classification scheme, there are clear systematic trends in the bulge-to-disk ratios, surface brightnesses and in the concentration of light, all of which increase from spirals to ellipticals. Star formation rates and gas content decrease along this same sequence.

With the advent of large spectroscopic surveys of nearby

* fabello@MPA-Garching.MPG.DE galaxies, such as the Sloan Digital Sky Survey (SDSS, York et al. 2000), the relationships between galaxy properties that can be derived from the combination of optical imaging and spectroscopy, e.g. stellar mass, size, concentration index, star formation rate, metallicity, dust content, have now been systematized and quantified in considerable detail (e.g., Kauffmann et al. 2003b; Brinchmann et al. 2004; Tremonti et al. 2004; Baldry et al. 2004, 2006). Our understanding of how the neutral gas content of galaxies relates to other galaxy properties lags far behind.

The cold gas content of a galaxy is known to vary strongly with colour and star formation rate. The connection between gas content and galaxy morphological type remains unclear. Whereas star-forming spiral galaxies almost always contain $\mathrm{HI}$ gas, the Hi content of early-type galaxies 
is considerably more difficult to predict. Some Es and S0s have neutral atomic hydrogen gas content similar to those of Sb-Sc type spirals, while others contain several orders of magnitude less Hi (Roberts \& Havnes 1994). It has been speculated that these variations may be an indication that the Hi gas in ellipticals has an external origin (Knapp et al. 1985), but direct proof of this conjecture is still lacking.

Because the HI is on average more difficult to detect in early-type galaxies, the samples discussed in the literature have generally been quite small. Some of the largest systematic studies of gas in early-type galaxies were carried out in the 1980's. Knapp et al. (1985) analyzed a sample of 152 nearby elliptical galaxies, of which 23 were detected in the Hi line. These authors studied the distribution of the quantity $\mathrm{M}_{\mathrm{HI}} / \mathrm{L}_{B}$ for these systems. In contrast to spiral galaxies, where the distribution $\mathrm{N}\left(\mathrm{M}_{\mathrm{HI}} / \mathrm{L}_{B}\right)$ has a well-defined mean value and a small dispersion, $\mathrm{M}_{\mathrm{HI}} / \mathrm{L}_{B}$ spans a wide range in ellipticals. Wardle \& Knapp (1986) extended this work to S0 galaxies and found that the Hi detection rate was twice as high compared to ellipticals. Some years later, Bregman et al. (1992) carried out a study of the interstellar components of 467 early-type galaxies in the Revised Shapley Ames Catalogue and again reported a trend of increasing neutral gas content from E to Sa. These authors suggested that the cold gas in early-types is associated mainly with disks and not with the bulge components of these galaxies. However, more recent studies that have mapped $\mathrm{HI}$ in nearby early-type galaxies (Morganti et al. 2006) have concluded that the Hi can be organized in a variety of different configurations, e.g. in regular disks, in clouds, in rings, or even in tidal tail-like structures.

One major problem that has plagued our understanding of cold gas in early-type galaxies is that the available Hi data have been inhomogeneous. Large area, blind Hi surveys such as the Arecibo Legacy Fast ALFA Survey (ALFALFA; Giovanelli et al. 2005) offer uniform coverage over large regions of the sky and allow one to construct complete, unbiased samples of HI-selected galaxies. However, these surveys are shallow, and do not in general detect gaspoor early-type galaxies. This limitation has been pointed out in recent papers by di Serego Alighieri et al. (2007) and Grossi et al. (2009), which used ALFALFA data to study an unbiased sample of early-type galaxies in the Virgo cluster region. They were able to compare the Hi content of earlytype galaxies drawn from field and group environments, but their average detection rates were much smaller than earlier studies based on incomplete and inhomogeneous data.

The Hubble morphological classification scheme is based on the optical appearance of a galaxy which in turn depends both on structural properties such as its bulge-todisk ratio, and on star formation rate. If we wish to understand the physical processes that regulate the neutral gas content of galaxies, it is preferable to analyze the effects of star formation and galaxy structure separately. Recently, Helmboldt (2007) studied $30 \mathrm{E}$ and S0 galaxies with signs of recent or ongoing star formation and concluded that such systems are more gas rich than E/S0 galaxies with old stellar populations. The availability of sizes, surface brightnesses and parameters measuring the concentration of the light for samples of millions of galaxies made possible due to recent advances in large scale CCD surveys and automatic image processing techniques, enable a new approach to un- derstanding the interplay between stars and gas in earlytype systems.

In this paper, we make use of stacking techniques to analyze whether the average Hi gas fraction of a galaxy is affected by the presence of a significant bulge component. Stacking has now become a common tool to constrain the statistical properties of a population of objects that lack individual detections in a survey; by co-adding the signal from many objects with known sky positions and redshifts, the background noise can be decreased and one can recover the average Hi flux of the ensemble. Stacking techniques have been applied to a wide variety of different astrophysical data. Examples include studies of faint radio AGN (Hodge et al. 2009), studies of star formation in high redshift Lyman Break Galaxies (Carilli et al. 2008), studies of the intracluster light using stacked optical images (e.g., Zibetti et al. 2005), and the soft X-ray properties of high redshift quasars (Shen et al. 2006). Stacking has been applied to Hi data as well, with the purpose of studying the Hi properties of gas at redshifts that are currently not well probed by existing radio telescopes (individual detections of Hi emission reach redshifts $\mathrm{z} \sim 0.25$ and require extremely long integrations, Catinella et al. 2008). Chengalur et al. (2001) stacked non-detections in different regions of a $\mathrm{z}=0.06$ cluster to investigate environmental effects. More recently, Hi stacking was used by Verheijen et al. (2007) to probe the ButcherOelmer effect at $\mathrm{z} \sim 0.2$, and by Lah et al. (2007, 2009) to attempt to constrain the Hi content of star-forming galaxies at $\mathrm{z} \sim 0.24$ and galaxies around a cluster at $\mathrm{z} \sim 0.37$.

Here, we use ALFALFA survey data to constrain the average Hi gas fractions of an unbiased sample of massive early-type galaxies. We study how the Hi content depends on parameters such as stellar mass, stellar mass surface density, concentration index, central velocity dispersion and UV/optical colour. The paper is structured as follows. In section 2 we describe the samples considered in this paper. The stacking analysis is described in section 3 . First we study Hi gas fraction scaling relations for a complete sample of galaxies and then we compare our results with previous work (section 4). The analysis of the Hi properties of early-type galaxies is presented in section 5. All distancedependent quantities in this work are computed assuming $\Omega_{m}=0.3, \Lambda=0.7$ and $H_{0}=70 \mathrm{~km} \mathrm{~s}^{-1} \mathrm{Mpc}^{-1}$.

\section{SAMPLE SELECTION}

Measured redshifts are essential if we wish to recover an accurate estimate of the mean Hi content of a population of galaxies using stacking techniques. Our sample is drawn from the "parent sample" of the GALEX Arecibo SDSS Survey (Catinella et al. 2010, hereafter GASS-1), which is a sample of 12006 galaxies with stellar masses greater than $10^{10} M_{\odot}$ and redshifts in the range $0.025<$ $z<0.05$ selected from the SDSS main spectroscopic sample. The parent sample galaxies are located in the intersection of the footprints of the Data Release 6 of the SDSS (DR6; Adelman-McCarthy et al. 2008), the projected GALEX Medium Imaging Survey (MIS; Martin et al. 2005) and ALFALFA. The average uncertainty in the SDSS spectroscopic redshifts is extremely small (0.0002). As discussed in GASS-1 (see section 3 for further details), the stel- 

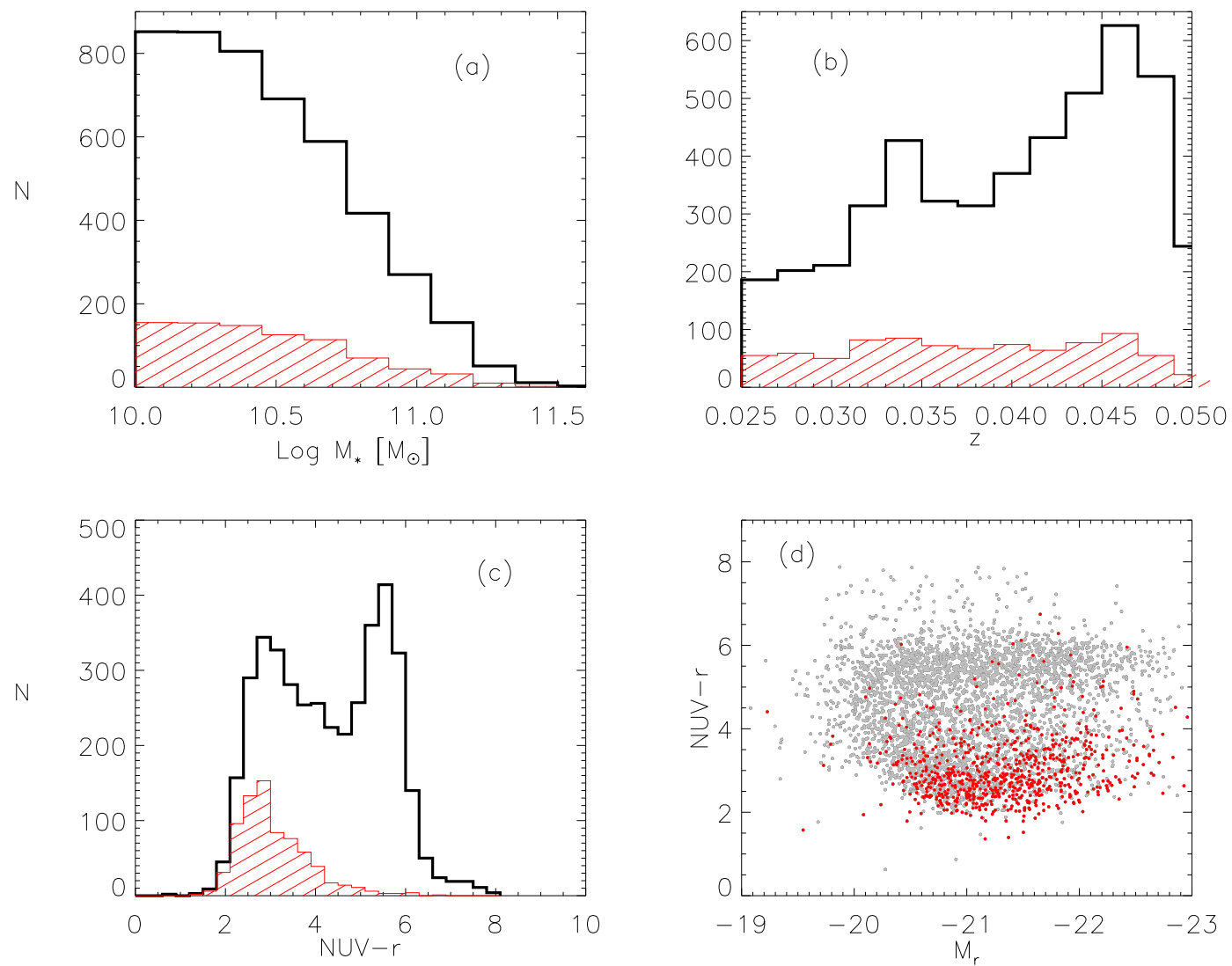

Figure 1. Distributions of (a) stellar mass, (b) redshift and (c) NUV-r colour (corrected for Galactic extinction only) for galaxies in sample $A$. The black solid histograms represent the whole sample $A$, while the red, dashed histograms show the distributions for the sub-sample of galaxies with ALFALFA detections. Panel (d) shows the colour-magnitude diagram of sample A galaxies (gray dots) and the sub-sample with ALFALFA detections (red dots).

lar masses are derived from SDSS photometry following Salim et al. (2007) and have typical errors smaller than $30 \%$. The choice of redshift range is determined by sensitivity limits in the Hi observations and by the need to avoid redshift ranges where radio frequency interference (RFI) is a problem.

The acquisition of ALFALFA data is on-going. Part of the data have already been catalogued and are available to the public (for the ALFALFA sky covered by SDSS: Giovanelli et al. 2007; Kent et al. 2008; Stierwalt et al. 2009). The ALFALFA 40\% dataset to be released in late 2010 (Martin et al. in preparation; Giovanelli et al., in preparation) includes the following SDSS sky regions: $7.5 \mathrm{~h}<$ $\alpha_{2000}<16.5 \mathrm{~h},+4^{\circ}<\delta_{2000}<+16^{\circ}$ and $+24^{\circ}<\delta_{2000}<$ $+28^{\circ}$, and $22 \mathrm{~h}<\alpha_{2000}<3 \mathrm{~h},+14^{\circ}<\delta_{2000}<+16^{\circ}$.

Within the same sky region, the GASS sample contains 5350 galaxies; these constitute the parent sample for our study. We discard some objects because they have poor quality Hi data (see section 3.1), so that the final GASS-SDSSALFALFA sample (which we call sample A) is composed of 4726 objects. Of these, $23 \%$ are galaxies with reliable ALFALFA detections (i.e. objects corresponding to ALFALFA detection codes 1 or 21 ). Panels (a), (b) and (c) of Fig-

1 As discussed in Giovanelli et al. (2005), ALFALFA HI line de- ure 1 show the stellar mass, redshift and NUV-r colour distributions of the galaxies in sample A. The NUV-r colours have been corrected for foreground Galactic extinction, but not for internal extinction. The ALFALFA detection rate is close to $23 \%$ for each stellar mass bin in Figure 1 but is clearly biased to blue-sequence objects (with NUV $-r \lesssim 3.5$ ) as shown in panel (c). Finally, in panel (d) we plot NUV-r colour versus absolute $r$-band magnitude $M_{r}$ for the sample, with black dots representing the full sample and red points the galaxies detected by ALFALFA. Once again we see that the galaxies detected by ALFALFA are almost exclusively found on the blue sequence.

\subsection{Galaxy Parameters}

The optical parameters we use are derived from the MPAJHU SDSS DR7 release of spectrum measurements or from Structured Query Language (SQL) queries to the SDSS DR7

tections are coded into two categories: Code 1 detections have a peak signal-to-noise ratio greater than 6.5 and are reliable at greater than 95\% confidence; Code 2 detections, referred to as "priors", have a lower signal-to-noise ratio between 4.5 and 6.5 but an optical counterpart at the same known redshift. Their reliability is estimated to be greater than $85 \%$. 

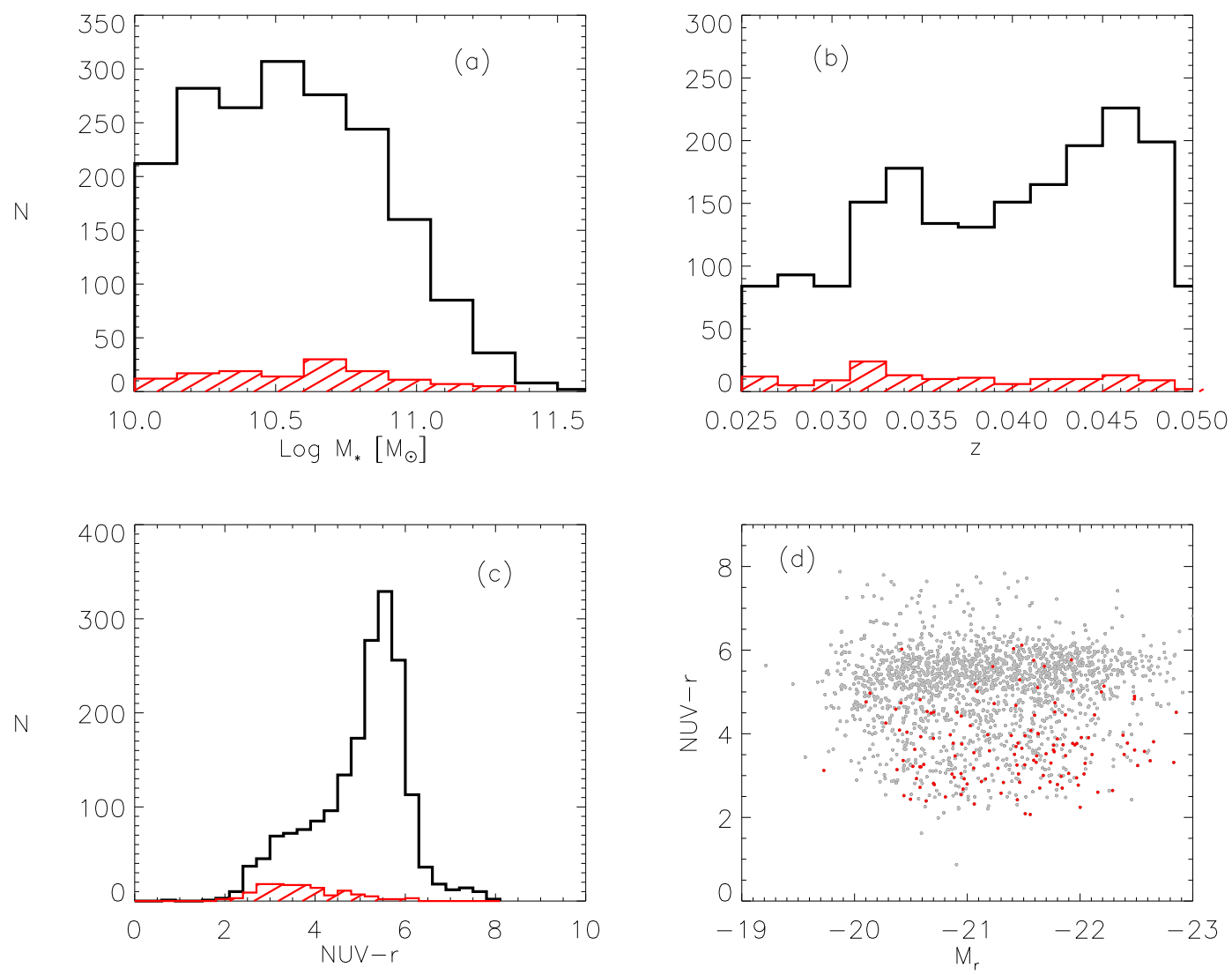

Figure 2. As in Figure 1, except for the ETG sample.

database server $2^{2}$. The UV parameters are extracted from the GALEX UV photometry by Wang et al. (2009). The reader is referred to section 5 of GASS- 1 for more detailed descriptions.

The parameters used in this paper are the following: 1) stellar mass $\left.M_{\star}, 2\right)$ stellar mass surface density $\mu_{\star}$, defined as $\mu_{\star}=\mathrm{M}_{\star} /\left(2 \pi R_{50, z}^{2}\right)$, where $R_{50, z}$ is the Petrosian radius containing $50 \%$ of the flux in $z$-band in units of $\mathrm{kpc}, 3)$ the concentration index $C=R_{90} / R_{50}$, where $R_{90}$ and $R_{50}$ are the radii enclosing $90 \%$ and $50 \%$ of the $r$-band Petrosian flux, 4) and NUV-r colour. As already explained, the colour is corrected for Galactic extinction only. Corrections for internal dust-attenuation are discussed and applied in Schiminovich et al. (2010), where they study the star formation properties of the GASS sample.

In addition to these quantities, we extract the following photometric parameters:

(i) The inclination $i$ to the line-of-sight is evaluated according to: $\cos i=b / a$, where $a$ and $b$ are the semi-major and semi-minor axes from the $r$-band exponential fit, respectively ( $b / a$ for the exponential fit is tabulated as $\exp A B_{\_} R$ ).

(ii) The likelihood parameters (lnldev_r and lnlexp_r from SDSS), which indicate how well the de Vaucouleurs and the

${ }^{2}$ See http://www.mpa-garching.mpg.de/SDSS/DR7/ and http://cas.sdss.org./dr7/en/tools/search/sql.asp exponential models fit the one-dimensional $r$-band light profile of the galaxy

(iii) The central velocity dispersion $\sigma_{1 / 8}$ is derived from the SDSS parameter vdisp. These velocities are evaluated with the "direct fitting" method ${ }^{3}$ using spectra measured within the $3^{\prime \prime}$-diameter fiber aperture. Only values between 70 and $420 \mathrm{kms}^{-1}$ are reliable. We then correct vdisp for aperture effects. Following Graves et al. (2009a) and references therein, we scale the fiber velocity dispersion to that at $1 / 8$ of the effective radius: $\sigma_{1 / 8}=v \operatorname{disp} \cdot\left(r_{f i b} / \frac{1}{8} r_{\circ}\right)^{0.04}$, where $r_{f i b}=1^{\prime \prime} .5$ and $r_{\circ}$ is the circular galaxy radius, defined as $r_{\circ}=R_{e} \sqrt{b / a}$. $R_{e}$ is the r-band de Vaucouleurs radius (tabulated as devR_r), and $a, b$ are now the major and minor axis from the de Vaucouleurs fit $(b / a$ is tabulated as devab_r). In general, this correction is small, $\sim 5 \%$. (Note that for galaxies included in sample $A, R_{e}$ has a mean value of $8.2^{\prime \prime}$.)

\subsection{ETG sample selection}

In this paper, we have chosen to define "early-type" galaxies purely in terms of their structural properties, without regard to their stellar populations or star formation rates. We note that our definition is in contrast to some definitions of

3 See http://www.sdss.org/DR6/algorithms/veldisp.html for more discussion 
"early-types" in the literature, which have excluded galaxies with emission lines (e.g., Bernardi et al. 2003; Graves et al. 2009a). Our goal will be to explore the extent to which the presence or absence of a significant bulge component influences the Hi content of a galaxy, so we do not wish to bias our conclusions by selecting against bulge-dominated galaxies with emission lines.

Starting from sample A, we extracted a subset of earlytype objects (ETG sample) with the following properties:

- concentration index $C=R_{90} / R_{50} \geq 2.6$;

- the likelihood that the light profile is fitted by a de Vaucouleurs model is greater than it is by an exponential one;

- inclination less than $70^{\circ}$ (this cut rejects the most inclined systems).

We note that the $C$ parameter has been shown to be an excellent indicator of the bulge-to-total ratio $(\mathrm{B} / \mathrm{T})$ derived from full 2-dimension bulge/disk decomposition analysis (Gadotti 2009). A cut at $C \geq 2.6$ restricts the sample to galaxies with $B / T \geq 0.4$ (see Fig.1 of Weinmann et al. 2009). For the present work we choose our default cut at $C \geq 2.6$, but we also experiment with cuts at larger values of $C$.

Application of our default cut leads to a final ETG sample consisting of 1833 objects. The properties of this sample are shown in Figure 2, The solid histograms represent the entire sample; the red dashed histograms the sub-sample detected by ALFALFA. The stellar mass, redshift and the NUV-r colour distributions are shown in panels (a), (b) and (c), respectively. The average ALFALFA detection rate for early-type objects is smaller $(\sim 9 \%)$ than for sample $A$. The colour-magnitude diagram is shown in panel $(d)$. Most of the early-type targets lie on a well-defined red sequence. Some objects do scatter bluewards of the red sequence. Such objects may be star-forming, transitional or Seyfert galaxies (Schawinski et al. 2007). By selecting targets based on concentration index and inclination, we may also include objects with some disk component. In Figure 3 we presents $1 \times 1$ arcminute SDSS postage stamps of a randomly selected set of galaxies from our ETG sample, that have been ordered by increasing $C$.

\section{ALFALFA DATA STACKING}

The on-going ALFALFA survey is scanning $7000 \mathrm{deg}^{2}$ of the high galactic latitude sky over the velocity interval $v\left[\mathrm{~km} \mathrm{~s}^{-1}\right] \simeq[-2500 ; 18000]$ (i.e. out to z 0.06) using a simple "minimum-intrusion" drift scanning technique (Giovanelli et al. 2005) that exploits the sevenhorn Arecibo L-band feed array (ALFA). For each of the seven beams, spectra are recorded separately for the two orthogonal polarizations, providing two independent samples. The 2-D drift scan data from both polarizations and all beams covering a given portion of the sky are combined to form 3 -D cubes of dimension $2.4^{\circ} \times 2.4^{\circ}$ on the sky and 5500 $\mathrm{km} \mathrm{s}^{-1}$ in velocity "depth". We refer the reader to Figure 4 where a schematic representation of one such data-cube is shown. We will refer to this depiction throughout the paper.

The raw spectral resolution of the ALFALFA data, before smoothing, is $\sim 5.5 \mathrm{~km} \mathrm{~s}^{-1}$ and the angular resolution is $\sim 3.3^{\prime} \times 3.8^{\prime}$ (corresponding to the FWHM of each ALFA beam). The data-cubes or "grids" are constructed from the drift scan data so that each spatial pixel is $1^{\prime}$ on a side. The 2-D drift scans are flux calibrated using the real-time noise diode injection scheme; the position and flux scales of the final grids are updated using fits to the many radio continuum sources they contain (Kent et al. 2008). The ALFALFA processing scheme (Giovanelli et al. 2005) retains all individually recorded spectra for each ALFA beam and each of its polarizations separately; no filtering is performed to discard bad channels. In order to deal with poor quality data, the 2-D data for each beam and polarization are visually inspected to flag bad records and frequency channels. The 3-D grid construction then proceeds with knowledge of the flagged 2-D pixels. Data quality in the final grid then may be limited by the absence of data in channels flagged as contaminated by RFI, weighted as poor by occasional instrumental problems or missing entirely because of incomplete sky coverage. In order to account for these effects, each 3-D pixel of the data-cube is assigned a quality weight $w$ during the data reduction process, which is a number ranging from 0 (unusable data) to 20 (good data), computed according to the availability and quality of data contributing to each pixel. The retention of the full dataset and the construction of the accompany "weights map" allows us to judge whether or not adequate data exist for each target so that it can be meaningfully included in the stacking process.

\subsection{Creating a catalogue of $\mathrm{HI}$ spectra}

We extract a spectrum for each galaxy in the sample. As discussed, all our targets are selected from the SDSS spectroscopic survey, so we know both their positions on the sky and their redshifts. We first select the ALFALFA data-cube which contains the target and then follow a procedure that includes the following steps: $a$ ) spectrum extraction; b) rms evaluation, $c$ ) final quality check.

\section{a. Spectrum extraction}

The signal from each target is integrated over a region of the data cube centered on its $3-\mathrm{D}$ position. Because noise increases with the square root of the integration area, integrating over too large a region lowers the quality of the spectrum without increasing the signal. Our GASS targets are always smaller then the ALFA beam (the mean $R_{90}$ for sample $A$ is $10^{\prime \prime}$ ), so we simply integrate over a sky region of $4^{\prime} \times 4^{\prime}$. In Figure 4 we illustrate how we extract spectra at two different positions in the sky inside the same data-cube. The coloured regions indicate where the spectra would be evaluated.

The Hi spectrum is a histogram of flux density $S$ as a function of velocity. For each velocity channel $v$, the corresponding flux density $S_{v}$ is obtained by integrating the signal $s_{v}(x, y)$ over the spatial pixels centered at the target galaxy position, as observed by a radio telescope of beam response pattern $\mathrm{B}$ :

$S_{v}[\mathrm{mJy}]=\frac{\Sigma_{x} \Sigma_{y} s_{v}(x, y)}{\Sigma_{x} \Sigma_{y} B(x, y)}$,

where $x, y$ are the sky coordinates (the two polarizations are kept separated). The expression above means that the spatially integrated profile is obtained by summing the sig- 

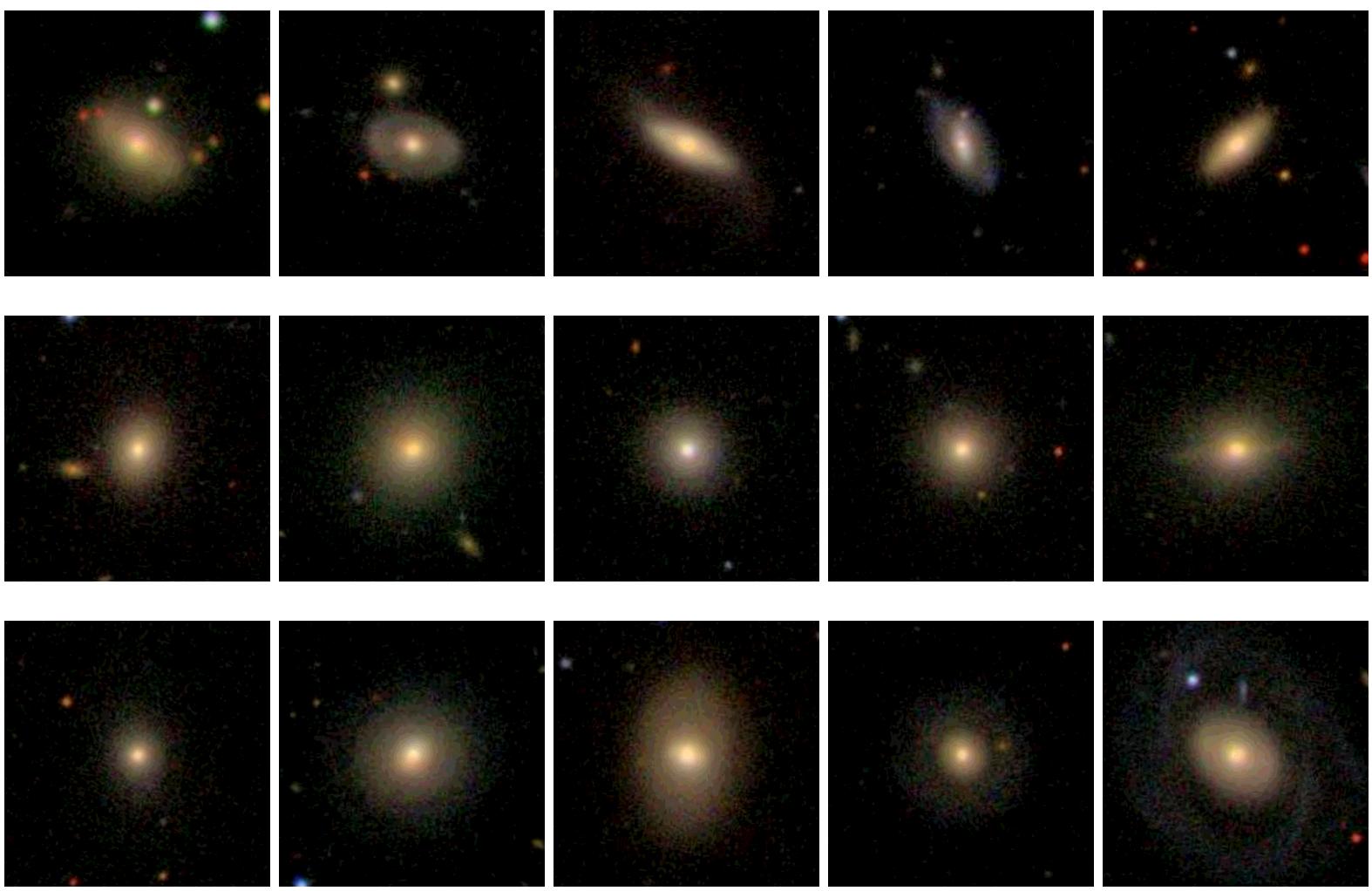

Figure 3. SDSS postage images of galaxies randomly selected from the ETG sample and ordered by increasing $C$. From left to right, top to bottom $C$ increases from 2.6 till 3.8. The images are 1 arcminute square in size.
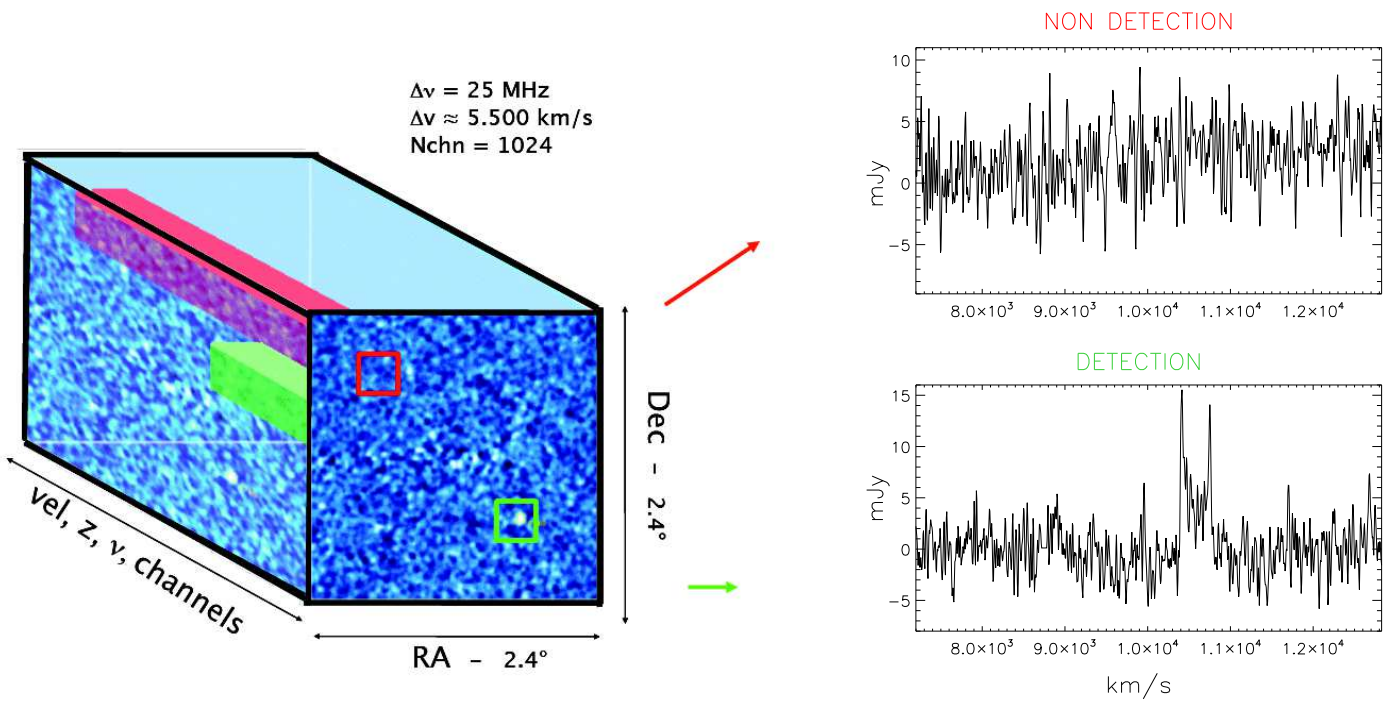

Figure 4. A schematic representation of the fully processed ALFALFA 3-D data-cube. The data cubes are $2.4^{\circ} \times 2.4^{\circ}$ in size and about $5500 \mathrm{~km} \mathrm{~s}^{-1}$ in velocity range (25 MHz in frequency). The raw spectral resolution is $\sim 5.5 \mathrm{~km} \mathrm{~s}^{-1}$; the angular resolution is $\sim 4^{\prime}$. For each pixel, which is a point in RA, Dec and velocity, a value of flux density is recorded. For each target in sample A, we extract a spectrum at a given position of the sky, over the velocity range of the data-cube which contains the source. Two examples of extracted spectra are shown on the right, illustrating an Hi detection (green, bottom) and an Hi non-detection (red, top).

nals over all the spatial pixels of interest and dividing by the sum of the normalized beam $B(x, y)$ over the same pixels (for a detailed discussion see Shostak \& Allen 1980). The
ALFALFA beam pattern can be approximated by:

$$
B(x, y)=\exp \left(-\frac{1}{2}\left(\frac{x}{\sigma_{x}}\right)^{2}-\frac{1}{2}\left(\frac{y}{\sigma_{y}}\right)^{2}\right)
$$


with $\sigma_{x}=(2 \sqrt{2 \ln 2})^{-1} \times 3.3^{\prime}$, and $\sigma_{y}=(2 \sqrt{2 \ln 2})^{-1} \times 3.8^{\prime}$ (Giovanelli et al. 2005).

We note that we discard any spectrum if more than $40 \%$ of the pixels have a a quality weight $w$ less than 10. We also keep track of the three strongest continuum sources in an area covering $40^{\prime} \times 40^{\prime}$ around each source; strong continuum sources can affect our spectra by creating standing waves.

\section{b. Rms evaluation}

For each spectrum we need to measure the root mean square (rms) noise, which will later be used as a weighting factor when we stack spectra. The rms has to be evaluated in regions of the spectrum where there is no emission from the target galaxy, and we also have to avoid spectral regions where there are any spurious signals (e.g. residual RFI that we failed to flag, or Hi emission from companion galaxies). In order to define the spectral region that might contain galaxy emission, we estimate its expected Hi width as follows.

The expected width of the Hi spectrum will depend on the rotational velocity of the galaxy as observed along the line-of-sight. We estimate the expected velocity $w_{T F ; o}$ for each target, using the the Tully-Fisher relation. Following Giovanelli et al. (1997b), we use the SDSS $i$-band magnitude ( $k$-corrected and corrected for Galactic and internal extinction as in equations 11 and 12 in Giovanelli et al. (1997a)) to estimate $w_{T F}$, and the measured inclination of the galaxy to derive $w_{T F ; o}$. We are aware that the Tully-Fisher relation does not hold for all morphological types and environments. We do not think this is a major issue, because these velocities are only used to estimate the region of the spectrum that should contain significant signal from the galaxy.

We then fit a first order polynomial to the baseline after excluding the region of the spectrum containing signal from the galaxy. This step allows us to eliminate possible gradients in the background. (The top right panel in Figure 4 shows an example of a spectrum where the baseline is tilted). We perform a robust polynomial fit over the regions of the spectrum with high values of the quality factor $w$ and then evaluate the rms about the fit over the same region of the spectrum.

The average rms for the whole sample (for each polarization) is $3.6 \pm 0.5 \mathrm{mJy}$. After averaging the two polarizations, an rms of $\sim 2.5 \mathrm{mJy}$ is obtained; this is comparable to the average rms of $2.2 \mathrm{mJy}$ evaluated for published, reduced ALFALFA spectra.

\section{c. Final quality check}

After we have extracted the spectra, we visually inspect each of them. We check the extraction process, and we discard spectra with bad baselines caused by continuum sources and those with possible spurious signals close to the galaxy (e.g., if one polarization has a significantly stronger signal than the other, or if there is a strong signal close to the object, which may arise from a companion galaxy). These cuts eliminate 624 objects in sample $A$ ( $11 \%$ of the initial sample).

\footnotetext{
4 Standing waves are periodic fluctuations in the background which occur when radiation from a strong continuum source is multiply reflected and scattered by the telescope structure before reaching the receiver.
}

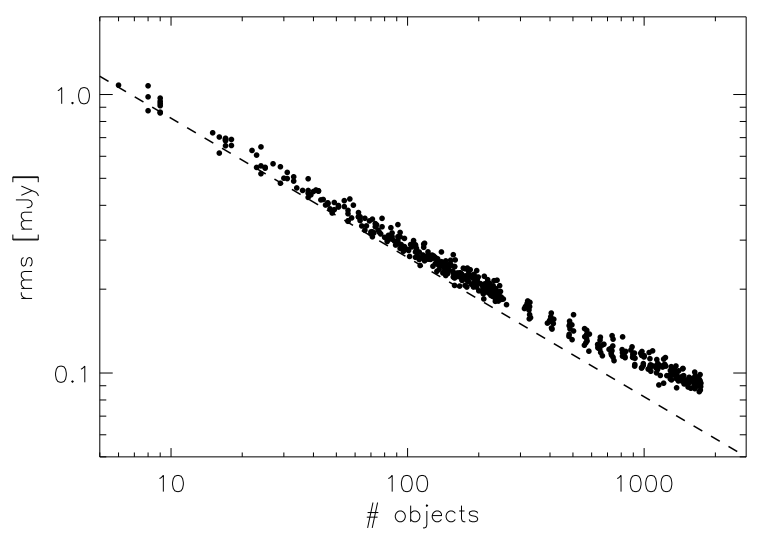

Figure 5. Dependence of the rms of the stacked spectra as a function of the number of objects co-added. The dashed line is the expected $1 / \sqrt{N}$ dependence. The flattening of the relation around $N \sim 300$ arises, as expected, where the non-Gaussian noise becomes dominant.

\subsection{The Stacking Method}

We want to co-add the signals from $N$ different sources located at different redshifts. First we shift each spectrum to the galaxy rest frequency, so each spectrum is centered at zero velocity. We stack together the spectra $S_{i}(\mathrm{i}=1, . . \mathrm{N})$ using their rms as a weight, a standard approach in stacking analysis. In doing this, the final spectrum $S_{\text {stack }}$ would be:

$$
\begin{aligned}
S_{\text {stack }} & =\frac{\sum_{i=0}^{N} S_{i} \cdot w_{i}}{\sum_{i=0}^{N} w_{i}} \\
w_{i} & =\frac{1}{r m s_{i}^{2}} .
\end{aligned}
$$

The stacking of the spectra is done separately for each polarization. Note that ALFALFA is a blind survey which scans the sky uniformly, so the rms for most spectra are similar. If the noise of the input targets is purely Gaussian, the $\mathrm{rms}$ of the stacked spectrum is expected to decrease as $1 / \sqrt{N}$, where $N$ is the number of objects co-added. In reality, in addition to the Gaussian noise there are likely to be systematic components, for example standing waves (we discarded spectra dominated by standing waves, but a weak residual signal could still remain). Because of these additional noise sources, we expect the rms to approach a lower limit as the number of co-added spectra becomes very large.

In Figure 5 we show how the measured rms of the stacked spectra decreases as a function of the number of coadded objects. We have stacked increasing numbers of randomly selected galaxies from sample $A$, and for each stack we evaluated an rms as described above. These measurements are shown as dots, while the dashed line shows the expected average rms value assuming Gaussian noise, i.e. $2.5 / \sqrt{N}$ mJy. As expected, we see the trend flatten as $\mathrm{N}$ approaches values of around 300, where the non-Gaussian noise becomes dominant. The $r m s$ continues to decline as $\mathrm{N}$ increases, but at a slower rate.

After stacking, we manually process each ensemble spectrum. The reduction process includes the following steps: $i$ ) we average the two polarizations. ii) A default Hanning smoothing is performed on the spectrum. Depending 
on the signal-to-noise, we may apply a boxcar smoothing to decrease the noise if the signal is marginal. Note that we never average over more than nine channels (corresponding to $\Delta v \sim 50 \mathrm{~km} \mathrm{~s}^{-1}$ ). iii) Finally, the baseline is subtracted. We note that the baseline of a stacked spectrum is already almost flat, because the different noise features tend to cancel out when averaging many spectra.

Figure 6] shows some examples of stacked spectra, obtained by stacking sample $A$ galaxies in five different stellar mass intervals. The vertical axis in each panel shows flux density in mJy, while the horizontal one shows velocity in $\mathrm{km} \mathrm{s}^{-1}$. Since we shifted each object to the rest-frequency of each galaxy, the stacked signal is centered at zero velocity. For each stacked spectrum, the mass range and the number of objects stacked are reported, as well as the signalto-noise ratio of the detected Hi signal. As explained above, when the signal-to-noise is low, we smooth the spectrum over up to nine channels as done, for example, for the last spectrum in Figure 6] In the left column of the Figure, we show spectra obtained by stacking ALFALFA detections and non-detections. In the right panel we show the spectra obtained by stacking only the non-detections, demonstrating that the stacking process recovers a signal even if no individual galaxy is detected (dotted lines in Fig. 6] indicate the edges of the signal). Notice that the rms decreases with increasing number of co-added objects. The width of the profile is smaller for less massive objects, as expected since they have on average lower circular velocities.

If we recover a signal in the stacked spectrum, we measure the integrated emission between the two edges of the Hi profile (see dotted lines in Figure 6), which are defined manually for each spectrum. We expect the signal to be symmetric around zero velocity. Even if in some cases the SDSS redshift is slightly off-set with respect to the Hi emission, the discrepancy will be random and will cancel out when co-adding multiple spectra (Note that the SDSS redshift uncertainty is 0.0002 , which corresponds to $\sim 60 \mathrm{~km} \mathrm{~s}^{-1}$ ).

We evaluate a signal-to-noise ratio following Saintonge (2007). We define an Hi detection if the $\mathrm{S} / \mathrm{N}$ is greater than 6.5. If there is no detection, we evaluate an upper limit, assuming a $5 \sigma$ signal with a width of $300 \mathrm{~km} \mathrm{~s}^{-1}$, smoothing the spectrum to $150 \mathrm{~km} \mathrm{~s}^{-1}$. (The width of $300 \mathrm{~km} \mathrm{~s}^{-1}$ is chosen because it corresponds to the peak of the distribution of velocity widths for galaxies in the GASS survey.)

\subsection{Evaluating HI gas fractions}

Our aim in stacking spectra is to characterize the average Hi content of a given sample of galaxies, so we are interested in converting our recovered signal into an Hi mass and also into an average Hi gas fraction. For a single object, these two quantities are well defined. The Hi mass is evaluated using the Roberts (1963) formula:

$$
\frac{M_{H I}}{\mathrm{M}_{\odot}}=\frac{2.356 \times 10^{5}}{1+z}\left(\frac{D_{L}(z)}{\mathrm{Mpc}}\right)^{2}\left(\frac{S_{i n t}}{\mathrm{Jy} \mathrm{km} \mathrm{s}^{-1}}\right),
$$

where $D_{L}(z)$ is the luminosity distance and $S_{\text {int }}$ the integrated Hi line flux. A correction for Hi self-absorption is not applied to the Hi mass, as it is likely to be negligible (Havnes \& Giovanelli 1984, Appendix B). The HI gas fraction is simply defined as $M_{H I} / M_{*}$.
For $\mathrm{N}$ individual detections (each with a flux measurement), the average value of their gas fractions can be defined by the weighted mean value:

$\left\langle\frac{M_{H I}}{M_{*}}\right\rangle=\left(\Sigma_{i=0}^{N} \frac{M_{H I ; i}}{M_{* ; i}} \cdot w_{i}\right) /\left(\Sigma_{i=0}^{N} w_{i}\right)$

where the $w_{i}$ are defined as in equation 3.2.

When co-adding spectra, we need to take into account the fact that our targets span a significant range in redshift $\left(z_{\max }=2 \cdot z_{\min }\right)$, so given the same Hi mass an object at the lowest redshift limit contributes 4 times more signal than one at the upper redshift limit. Because we are stacking mainly non-detected spectra we do not know how much each galaxy contributes to the total signal. Both the mean and median values of redshift and stellar mass may not be representative. In order to weight each spectrum in a consistent manner, we choose to stack "gas-fraction" spectra $S_{i}^{\prime}$, where the signal that we co-add is no longer the flux $S$ [mJy], but the quantity:

$S_{i}[\mathrm{mJy}] \rightarrow S_{i}^{\prime}\left[\mathrm{mJy} \mathrm{Mpc}^{2} \mathrm{M}_{\odot}^{-1}\right]=\frac{S_{i} \cdot D_{L}^{2}\left(z_{i}\right)}{M_{* ; i}}$

The stacking is performed according to equation 3.1. With this approach, the stacking creates an average object with respect to the Hi gas fraction of the individual galaxies used to build the stack. In Appendix A, we discuss an alternative approach to evaluating mean gas fractions from stacked spectra, and present comparisons between the two different methods.

We measure errors on the gas fractions using the Jackknife method (Tukev 1977; Efron 1982), a statistical tool to estimate a confidence interval on a function of $\mathrm{N}$ measures. The purpose of computing jackknife errors on the mean $\mathrm{HI}$ fraction estimated from a stacked spectrum is to ascertain whether or not the signal is dominated by a few outliers. Schematically, given a function $\hat{\alpha}\left(x_{i}\right)$ of $N$ observations $x_{i}$, the Jackknife evaluates $N$ partial estimates $\hat{\alpha}_{j}$ of the function obtained by discarding one element per time. The jackknifed estimate $\hat{\alpha}^{*}$ is then the average of the pseudo-values $\hat{\alpha}_{j}^{*}=N \hat{\alpha}-(N-1) \hat{\alpha}_{j}$. Finally, the sample variance on the pseudo-values is:

$$
\left(\sigma_{J a c k}\right)^{2}=\frac{1}{N(N-1)} \sum_{j=1}^{N}\left(\hat{\alpha}_{j}^{*}-\hat{\alpha}^{*}\right)^{2},
$$

This can be used to provide a confidence interval on $\hat{\alpha}-\hat{\alpha}^{*}$.

\section{Hi GAS FRACTION SCALING RELATIONS FOR MASSIVE GALAXIES}

In this section, we characterize how average Hi gas fraction depends on a variety of different properties for galaxies with stellar masses greater than $10^{10} M_{\odot}$. First we study the dependence on stellar mass $\mathrm{M}_{\star}$, stellar mass surface density $\mu_{\star}$, concentration index $C$ and colour NUV $-r$ for galaxies in sample $A$. We then compare the same relations for galaxies in the bulge-dominated ETG sample ( $(5)$.

We begin by comparing our results with those of Catinella et al. (2010), who studied the correlation between Hi gas fraction and the properties listed above, for a complete sample of $\sim 200$ galaxies from the GASS survey with $M_{*}>10^{10} M_{\odot}$. In that survey, the Hi observations reached 

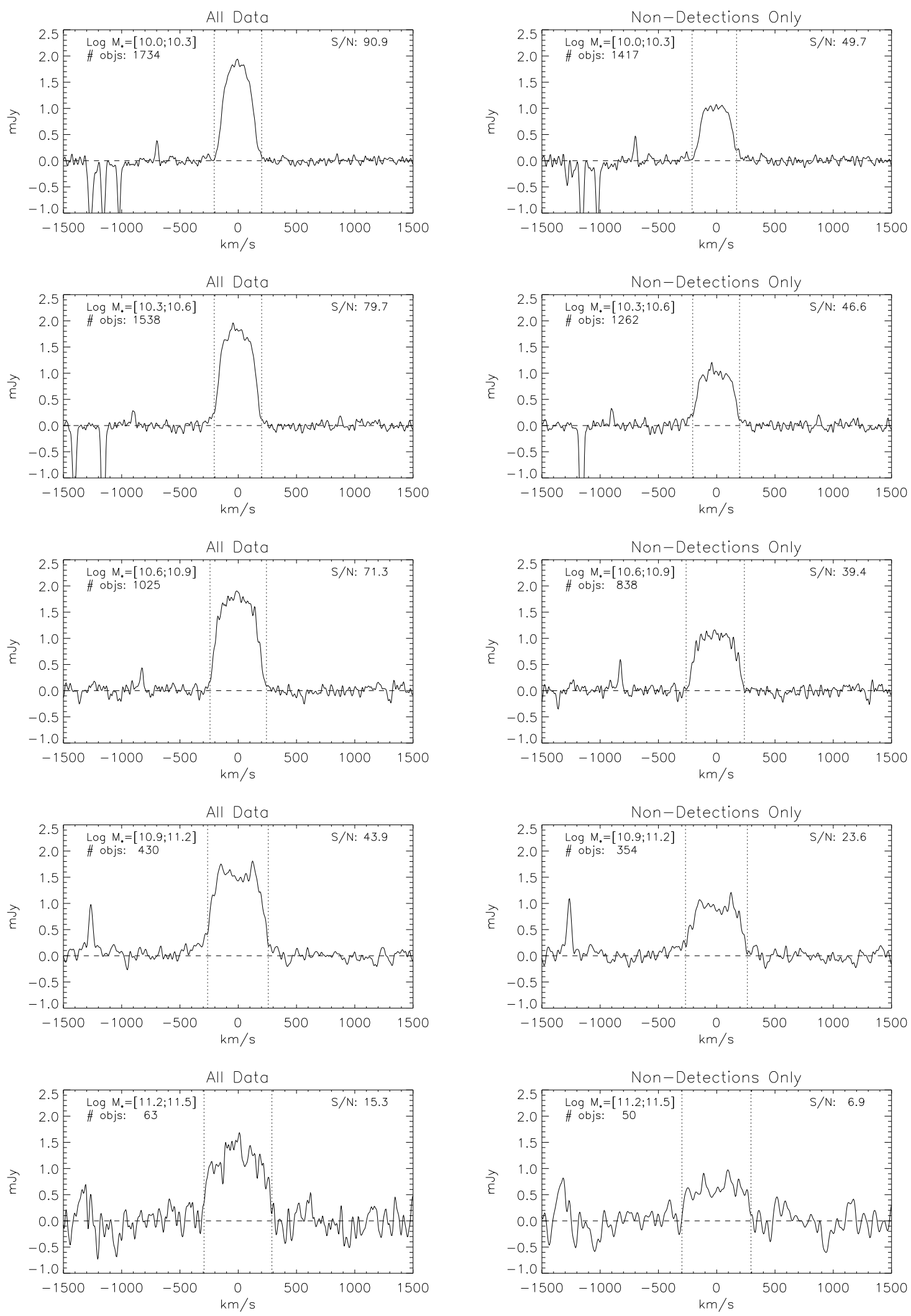

Figure 6. Examples of stacked spectra for $5 \mathrm{M}_{\star}$ bins. The $\mathrm{x}$-axis is velocity in $\mathrm{km} \mathrm{s}^{-1}$, the y-axis is Hi flux density in mJy. Since we shifted each object to its galaxy rest-frequency, the signal is centered at zero velocity. For each spectrum, the mass range and the number of objects stacked are reported, as well as its S/N ratio. Dotted lines show the boundaries of the signal, inside which we integrate the flux. In some spectra there are spikes/holes caused by poor quality data (note that the spectra containing the bad data were not discarded because the bad pixels are located away from the central regions of interest). Examples of bad regions occur in the first two rows between $v=-1500 \mathrm{~km} \mathrm{~s}^{-1}$ and $v=-1000 \mathrm{~km} \mathrm{~s}^{-1}$. Left column: stacked spectra using all galaxies. Right column: stacked spectra using only those galaxies that were not detected by ALFALFA. As expected, the signal is systematically lower for the right column. 

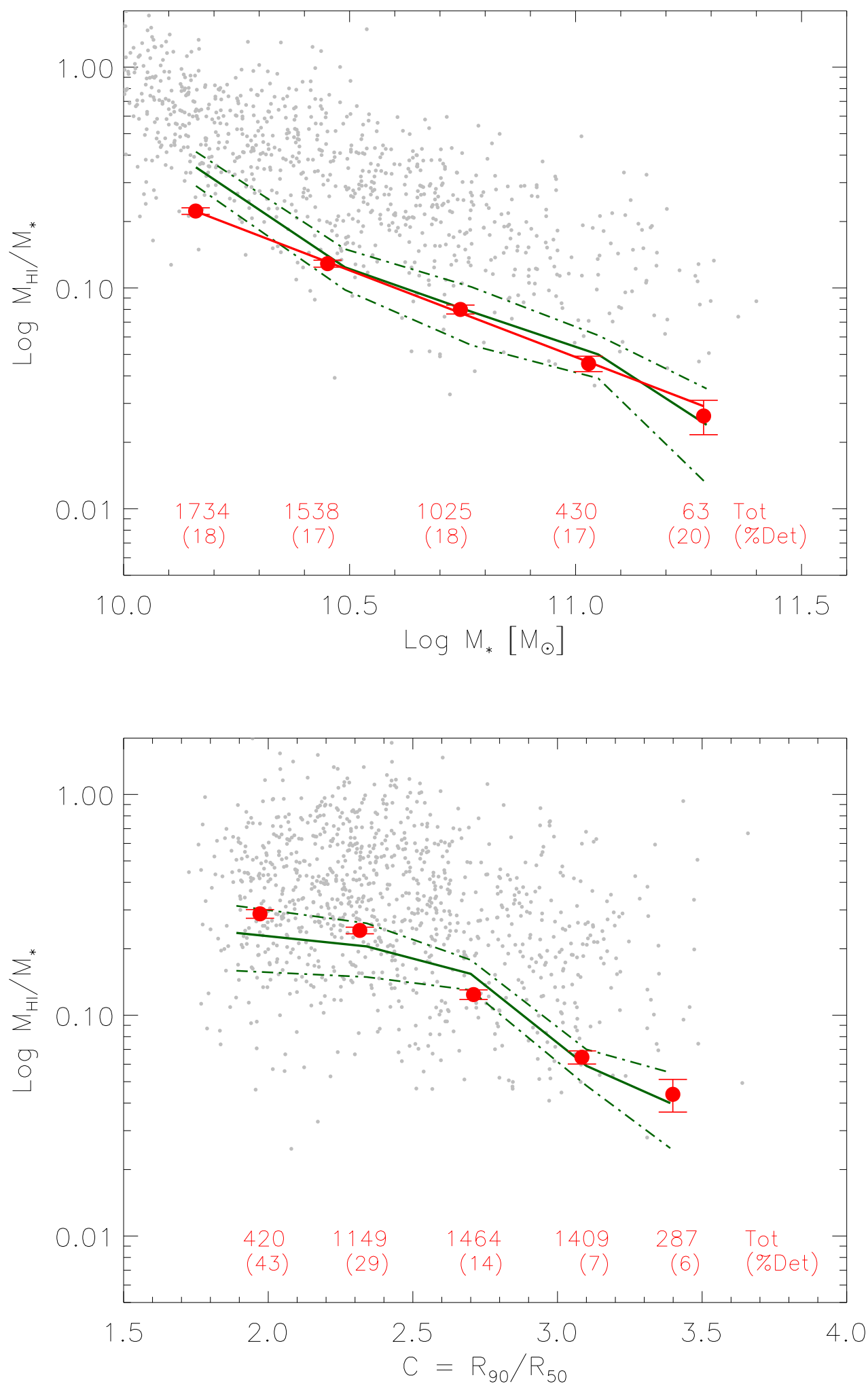

Figure 7. Red circles: Average gas fractions of sample $A$ galaxies derived from stacking are plotted as a function of stellar mass $M_{*}$ (top) and concentration index $C$ (bottom). The points are plotted at the mean value of $M_{*}$ or $C$ for the galaxies in the bin. The red line (upper panel) show a linear fit to these results, which are compared with the average Hi gas fractions from the GASS-1 paper (green lines). Green dashed lines show the $1 \sigma$ uncertainties on the GASS-1 estimates. Gray dots show galaxies with ALFALFA detections from sample $A$. The numbers written in the panels indicate the numbers of objects co-added in each bin (Tot), and the percentage of them directly detected by ALFALFA (\%Det). The values plotted are reported in Table A1 4th and 5th columns. 

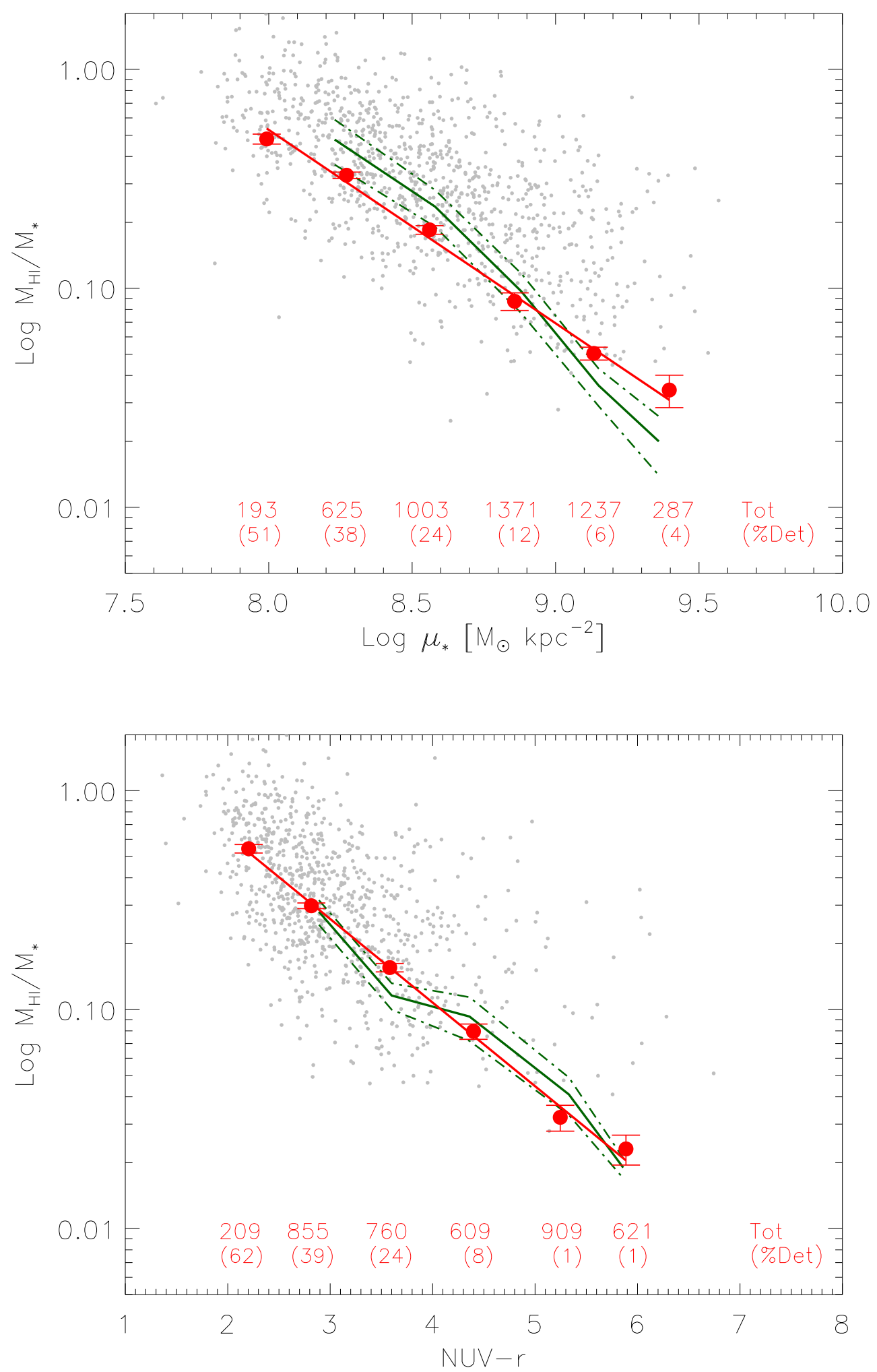

Figure 8. Average gas fractions of sample $A$ galaxies derived from stacking are plotted as a function of stellar mass surface density $\mu_{\star}$ (top) and NUV $-r$ colour (bottom). Symbols and colours as in Figure 7 The values plotted are reported in Table A1 4th and 5th columns. 
a much deeper flux limit compared with ALFALFA, so average Hi gas fractions could be derived using individual HI detections, rather than by stacking. It is important to check whether our stacking method gives answers that are consistent with these published measurements.

In Figures 7 and 8, we present our results for sample A. In each panel, gray dots show the galaxies in sample $A$ that were detected by ALFALFA. Green lines show the average values and $1 \sigma$ confidence interval of $\mathrm{M}_{\mathrm{HI}} / M_{*}$ from GASS-1 (see Figure 9 in that paper). Our own estimates of the HI gas fraction derived from the stacked spectra are plotted as red circles, and the errorbars on these points are evaluated using the Jackknife method (eq. 3.6). Note that the stacked spectra that yield the measurements plotted as red circles in Figure 7 (top panel), are shown in Figure 6 (left panel). At the bottom of each panel, we record the number of galaxies included in each stack, as well as the percentage of galaxies with ALFALFA detections. In cases where the stack includes more than a few hundred galaxies, the errors on the mean Hi fraction are negligible.

We note that we are able to measure an Hi gas fraction in every bin, even in the very reddest NUV-r colour bins where the ALFALFA detection rates are close to zero. The fact that the jackknife errors remain relatively small for these bins, indicates that the stacked spectra are not dominated by signal from a small fraction of the galaxies.

We find that the average Hi gas fractions derived from the stacked spectra are in excellent agreement with the results reported in GASS-1. The point-by-point agreement is generally within $1 \sigma$, with the possible exception of the lowest stellar mass and stellar mass surface density bins, where we find gas fractions that are systematically lower than those reported in the GASS-1 paper. We caution that low mass galaxies were somewhat under-represented in the first GASS data release. Our errorbars only indicate statistical errors and do not account for other effects, such as cosmic variance, so we do not think the discrepancy is significant.

As discussed in GASS-1, the Hi gas fraction is a decreasing function of stellar mass, stellar mass surface density, concentration index and colour. The slopes of these relations for our sample $A$ are listed in the 3rd column of Table A1. Here we only report the total change in $\log M_{\mathrm{HI}} / M_{*}$ $\left(\Delta \log M_{\mathrm{HI}} / M_{*}\right)$ for each relationship, which allows a comparison among the different properties:

$$
\begin{array}{rlll}
M_{*} & : & \Delta \log M_{\mathrm{HI}} / M_{*}=0.20 \\
C & : & \Delta \log M_{\mathrm{HI}} / M_{*}=0.24 \\
\mu_{*} & : & \Delta \log M_{\mathrm{HI}} / M_{*}=0.45 \\
\mathrm{NUV}-r & : & \Delta \log M_{\mathrm{HI}} / M_{*}=0.52
\end{array}
$$

The correlations between gas fraction and both $\mathrm{M}_{\star}$ and concentration index (Figure 17) are weak. In contrast, the average value of $M_{\mathrm{HI}} / M_{*}$ drops by more up to a factor of 25 when plotted as a function of NUV-r colour or stellar mass surface density. Although stacking recovers the average trends of the population extremely well, it does not provide any information on the underlying scatter. This can only be studied with individual gas fraction measurements, as in GASS-1.

\section{Hi STUDY OF A COMPLETE SAMPLE OF ETG GALAXIES}

In this section, we ask whether bulge-dominated galaxies with $M_{*}>10^{10} M_{\odot}$ lie on the same Hi scaling relations as the general population of galaxies with $M_{*}>10^{10} M_{\odot}$. Our goal is to determine whether the presence of the bulge plays a role in regulating the rate at which gas is consumed into stars, for example by stabilizing the disk (e.g., Martig et al. 2009). In order to test the role of the bulge in a clean way, we must account for the fact that the physical properties of galaxies are strongly correlated. If one selects a sub-sample of bulge-dominated galaxies from the parent sample $A$, one will automatically select a sample of galaxies with higher stellar masses, higher stellar mass surface densities, and redder colours. It is therefore important to understand whether or not bulge-dominated galaxies differ in HI content from the parent sample at fixed values of these parameters.

Our results are presented in Figures 9 and 10 and in Table A1 In all the plots, blue circles are the average gas fractions obtained from stacking the ETG sample and the errorbars are evaluated using the Jackknife method (eq.3.6. Upside-down triangles indicate the upper limit in the case of a stack that yields a non-detection. The red lines (circles) show the fits to the mean Hi gas fraction relations obtained for sample A. Our main result is that the average Hi gas fractions of bulge-dominated galaxies are significantly lower (by approximately a factor of 2) than those of the parent sample at a given value of stellar mass. A similar, but weaker reduction in the average $\mathrm{HI}$ gas fraction is seen for the ETG sample when it is plotted as a function of the central velocity dispersion of the bulge. However, the relation between gas fraction and stellar mass surface density and NUV-r colour appears to be insensitive to the ETG cut. In GASS-1 paper, Catinella et al. showed that a linear combination of stellar mass surface density and NUV-r colour provided an excellent way to "predict" the Hi content of galaxies more massive than $10^{10} M_{\odot}$. Here we show that this conclusion holds true independent of the bulge-to-disk ratio of the galaxy. We have tested that this conclusion still holds if we define the ETG sample using more stringent cuts on concentration index $(C>3)$ or on the axis ratio of the galaxy $(b / a>0.6$, which implies inclination lower than $\left.\sim 55^{\circ}\right)$. These cuts reduce the ETG by $50 \%$ and $30 \%$, respectively. Nevertheless, results shown in Figures 9 and 10 demonstrate that the average $\mathrm{HI}$ gas fractions of these systems still lie on the same relations when plotted as a function of stellar mass surface density and NUV-r colour (cyan circles represent the further cut in concentration index, black ones the cut in inclination).

In Figure 11] we show how the average gas fractions of galaxies in the ETG sample vary as a function of position in the two-dimensional plane of colour versus stellar mass density $\mu_{*}$. Bulge-dominated galaxies are mainly found on the red sequence, but there is a minority population with bluer colours. We adaptively bin the sample in two dimensions by recursively dividing the plane into axis-aligned rectangles. We stop dividing a region when a further split would lower the $\mathrm{S} / \mathrm{N}$ below the detection threshold of 6.5. Figure 11 (bottom panel) shows the final binning used. In each bin the measured gas fraction (expressed as a percentage of the stellar mass) is reported. In Figure 11 (top panel) we colourcode the $(\mathrm{NUV}-r)-\mu_{*}$ plane according to gas fraction. The 

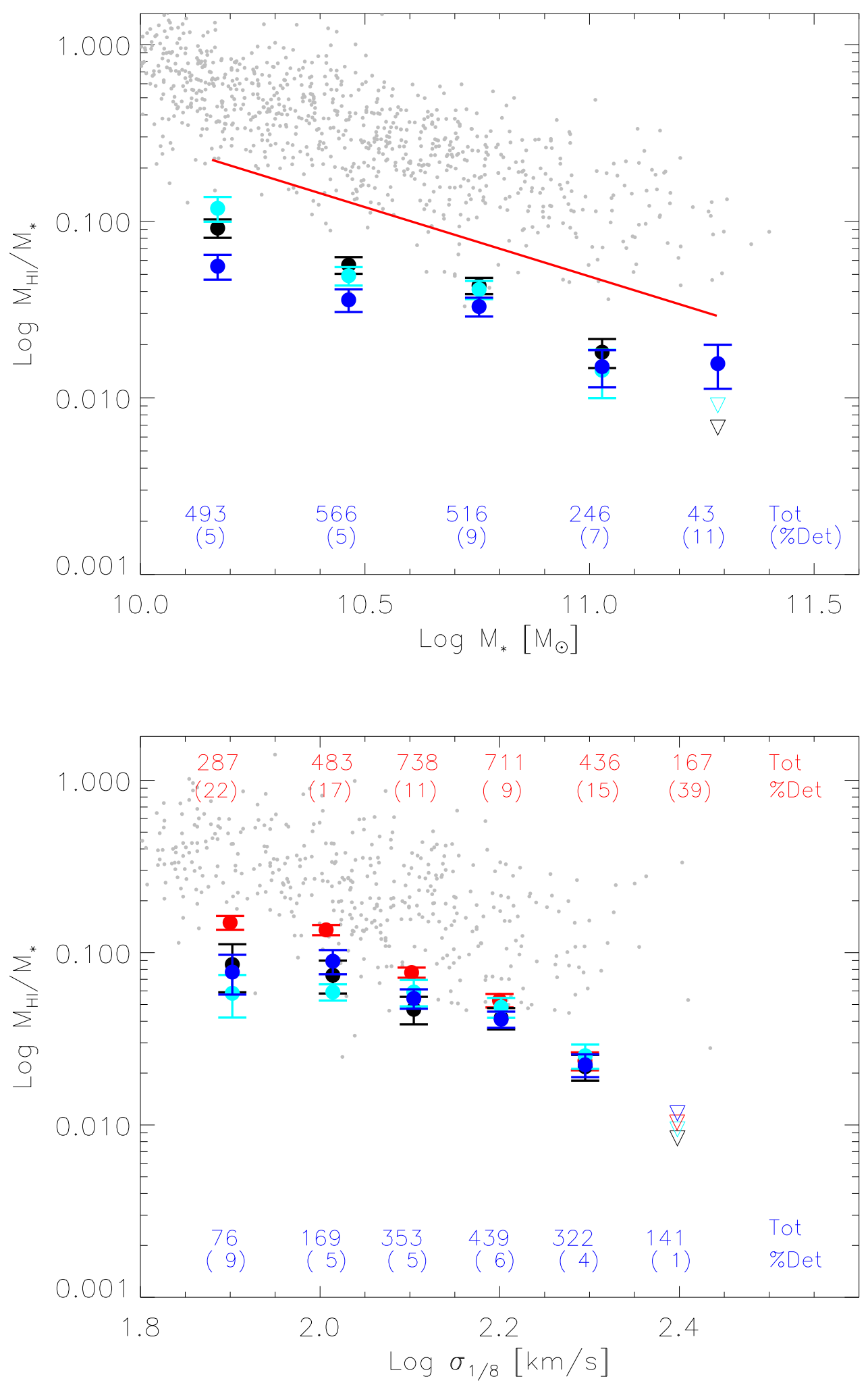

Figure 9. The dependence of the average Hi gas fraction on stellar mass $\mathrm{M}_{\star}$ (top) and on central velocity dispersion $\sigma$ (bottom) for the ETG sample (blue symbols). The relations found for sample $A$ are shown in red for comparison (the fit for the $\mathrm{M}_{\star}$ relation, the actual points for $\sigma$ ). Upside-down triangles indicate upper limits in the case of a non-detection. The numbers written in the panels indicate the numbers of objects co-added in each bin (Tot), and the percentage of them directly detected by ALFALFA (\%Det). Gray dots show sample A galaxies with ALFALFA detections. We have also applied more stringent cuts to the ETG sample, as explained in the text: cyan circles represent a sample with $C>3$ ( $52 \%$ of the original ETG sample); black circles are for a sample with b/a $>0.6$ (or inclination lower than $55^{\circ}-68 \%$ of the original sample). The values plotted for each ETG sample are reported in Table A1 6th-11th columns. 

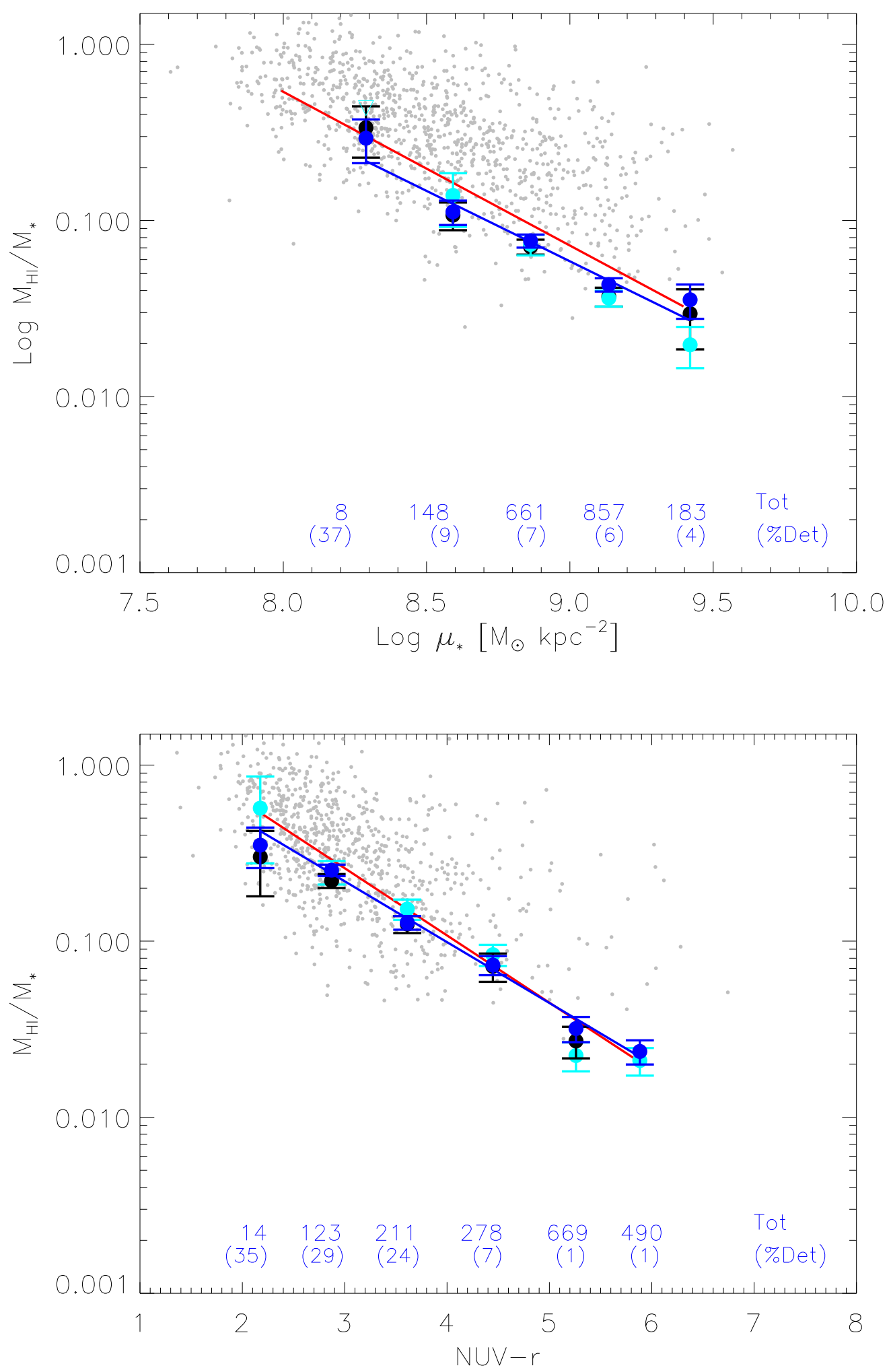

Figure 10. Average Hi gas fraction dependence on stellar mass surface density $\mu_{\star}$ (top) and NUV $-r$ colour (bottom) for the $E T G$ sample. Symbols and colours are the same as described in Figure 9 The values plotted for each ETG sample are reported in Table A1 6 th-11th columns. 

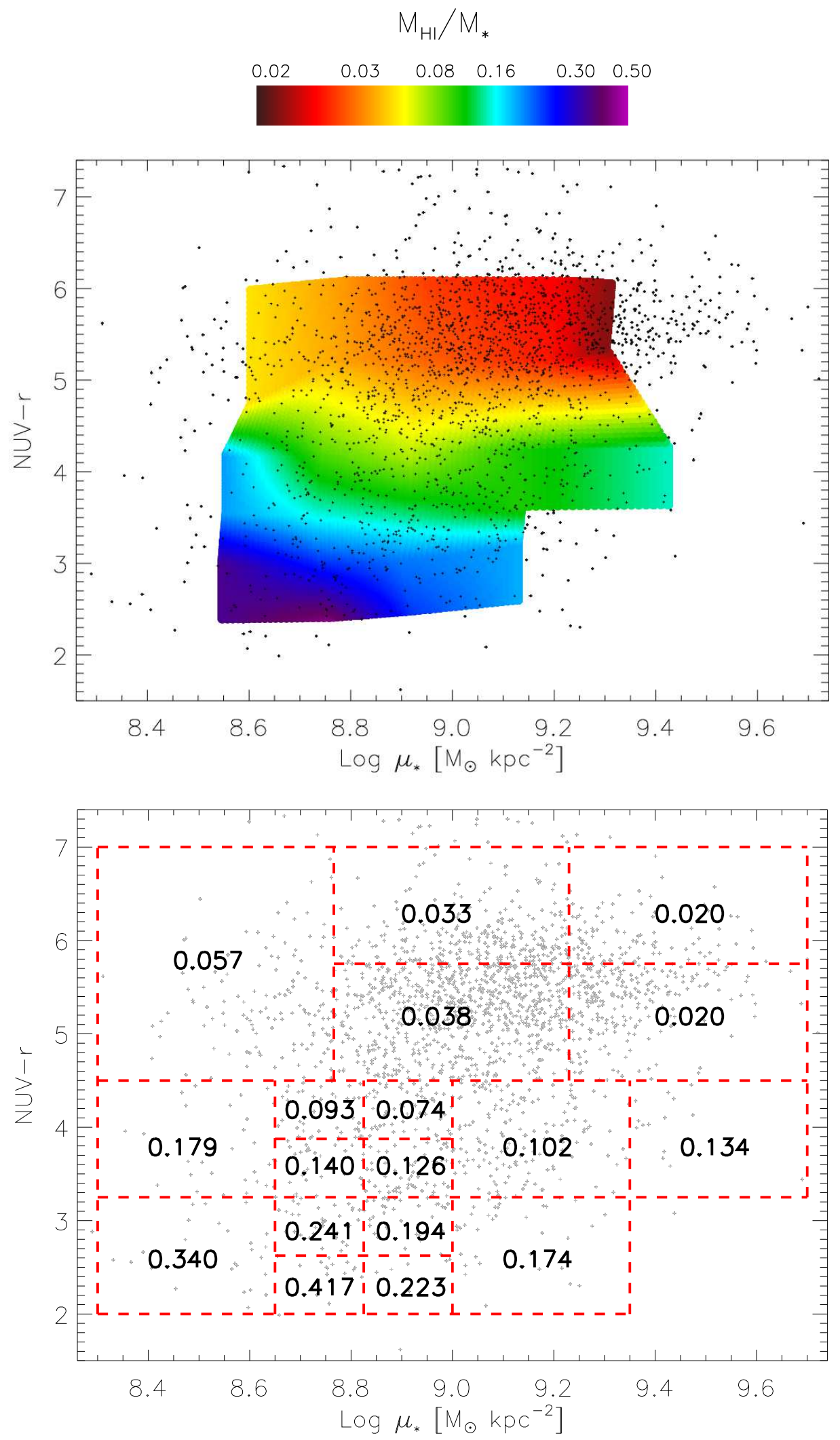

Figure 11. Average HI gas fraction dependence in the 2-dimensional plane of stellar mass surface density $\mu_{\star}$ and NUV-r colour for galaxies in the ETG sample. In the top panel the dots show individual objects, while the colors show the (interpolated) gas fractions measured with the stacking as a function of position in the plane (the colour scale key is included above the plot). The bottom panel shows the adopted binning. For each bin, the gas fraction measured from the stacked spectrum is noted. 
HI content decreases going from left to right (towards increasing stellar mass surface density) and from bottom to top (towards redder colours). The most significant variation is clearly along the colour direction.

\subsection{A Test of the morphological quenching scenario}

The idea that galaxy disks are more resistant to the formation of bars, spiral density waves and other instabilities if they are embedded within a dynamically hot halo or bulge, has its origins in early work by Ostriker \& Peebles (1973). Recently, Martig et al. (2009) have proposed this so-called "morphological quenching" mechanism as a way of explaining why present-day bulge-dominated galaxies on the red sequence cease growing in stellar mass in environments where they continue to accrete gas. In their picture, a disk with similar gas content will be much less efficient at forming stars if it is embedded in a galaxy with a significant bulge component. As stated in the abstract of their paper, "our mechanism automatically links the colour of the galaxy with its morphology, and does not require gas consumption, removal or termination of the gas supply."

To test whether the "morphological quenching" process is truly important in maintaining the low observed rates of star formation in red sequence galaxies, we have performed the following experiment. We have binned galaxies with $C<$ 2.6 and $C>2.6$ in the two-dimensional plane of NUV- $r$ colour versus stellar mass (note that we use the same bin boundaries for both samples). We stack the Hi spectra of the galaxies in each bin and calculate the average Hi gas fraction, as explained above. In Figure 12, we report for each bin the ratio between the gas fraction of the disk-dominated $(D D)$ objects and the bulge-dominated $(B D)$ ones, i.e.:

$$
r=\left(\frac{M_{H I}}{M_{*}}\right)_{D D} /\left(\frac{M_{H I}}{M_{*}}\right)_{B D}
$$

If the morphological quenching scenario is correct, then at fixed stellar mass, we would expect to find higher average HI gas fractions for bulge-dominated galaxies on the red sequence than for disk-dominated galaxies on the red sequence.

The results in Figure 12 show that in general the $o p$ posite is true. Gas fractions are always slightly higher for the disk-dominated galaxies than for bulge-dominated ones. The gas fraction differences do appear to be largest for red sequence galaxies with NUV-r $>5$, but the sign of the difference contradicts the predictions of Martig et al. (2009).

It is important to check that our result is not simply due to extinction effects. Some of the reddest, gas-rich diskdominated objects may actually be heavily obscured systems that will move blueward when dust corrections are applied. Following Schiminovich et al. (2010) we have applied dust corrections to the NUV-r colours of the galaxies in our sample with $\mathrm{D}_{n}(4000)<1.75$. In order to make up for the loss of red late-type galaxies, we had to apply stronger cut in concentration index $(\mathrm{C}>3)$ to define the early-type sample.

$5 \mathrm{D}_{n}(4000)$ is defined as the ratio of the average flux density in the continuum bands $3850-3950$ and $4000-4100 \AA$, and traces the age of stellar populations (Kauffmann et al. 2003a).
The ratio of gas fraction between disk-dominated and bulgedominated objects decreases, but we still find that the $\mathrm{HI}$ gas fraction of the disk-dominated galaxies never falls below that of the bulge-dominated ones. In particular, if we divide the red sequence objects with $4.5<\mathrm{NUV}-r<7$ in two bins of stellar mass, we find a value of $r=1.03$ for the less massive galaxies, and $r=1.71$ for the more massive ones.

We note that these results are in agreement with those presented in Schiminovich et al. (2010). In their paper, Schiminovich et al. use a volume-limited sample of 200 galaxies from the GASS survey to explore the global scaling relations associated with the ratio $\mathrm{SFR} / \mathrm{M}_{\mathrm{HI}}$, which they call the HI-based star formation efficiency. They found that the average value of this star formation efficiency has little variation with any galaxy parameter, including the concentration index.

\section{SUMMARY}

We have carried out a stacking analysis using ALFALFA scans of a volume-limited sample of $\sim 5000$ galaxies with imaging and spectroscopic data from GALEX and the Sloan Digital Sky Survey. The galaxies have stellar masses greater than $10^{10} M_{\odot}$ and redshifts in the range $0.025<z<0.05$. We extract a sub-sample of 1833 "early-type" galaxies with inclinations less than $70^{\circ}$, with concentration indices $C>$ 2.6 , and with light profiles that are well fit by a De Vaucouleurs model. We then stack the ALFALFA spectra of the galaxies from these two samples in bins of stellar mass, stellar surface mass density, central velocity dispersion, and NUV- $r$ colour, and we use the stacked spectra to estimate the average $\mathrm{HI}$ gas fractions $\mathrm{M}_{\mathrm{HI}} / M_{*}$ of the galaxies in each bin.

Our main result is that the HI gas fractions of both early-type and late-type galaxies correlate primarily with NUV- $r$ colour and stellar mass surface density. The relation between average Hi gas fraction and these two parameters is independent of $C$, and hence of the bulge-to-disk ratio of the galaxy. We note that at fixed stellar mass, early-type galaxies do have lower average Hi fractions than late-type galaxies, but this effect does not arise as a direct consequence of the presence of the bulge and we discuss possible implications below.

We have also tested whether the average Hi gas content of bulge-dominated galaxies differs from that of latetype galaxies at fixed values of NUV $-r$ and $\mu_{\star}$. We find no evidence that red-sequence galaxies with a significant bulge component are less efficient at turning their available gas reservoirs into stars. This result is in contradiction with the "morphological quenching" scenario proposed by Martig et al. (2009).

\section{DISCUSSION}

We now consider possible implications of this work.

1. The Hi content of a galaxy is independent of its bulge-to-disk ratio. This can be understood if the following two conditions are satisfied: 1) The Hi gas in earlytype galaxies is always associated with disks, or with material that is in the process of settling into disks. 2) The for- 


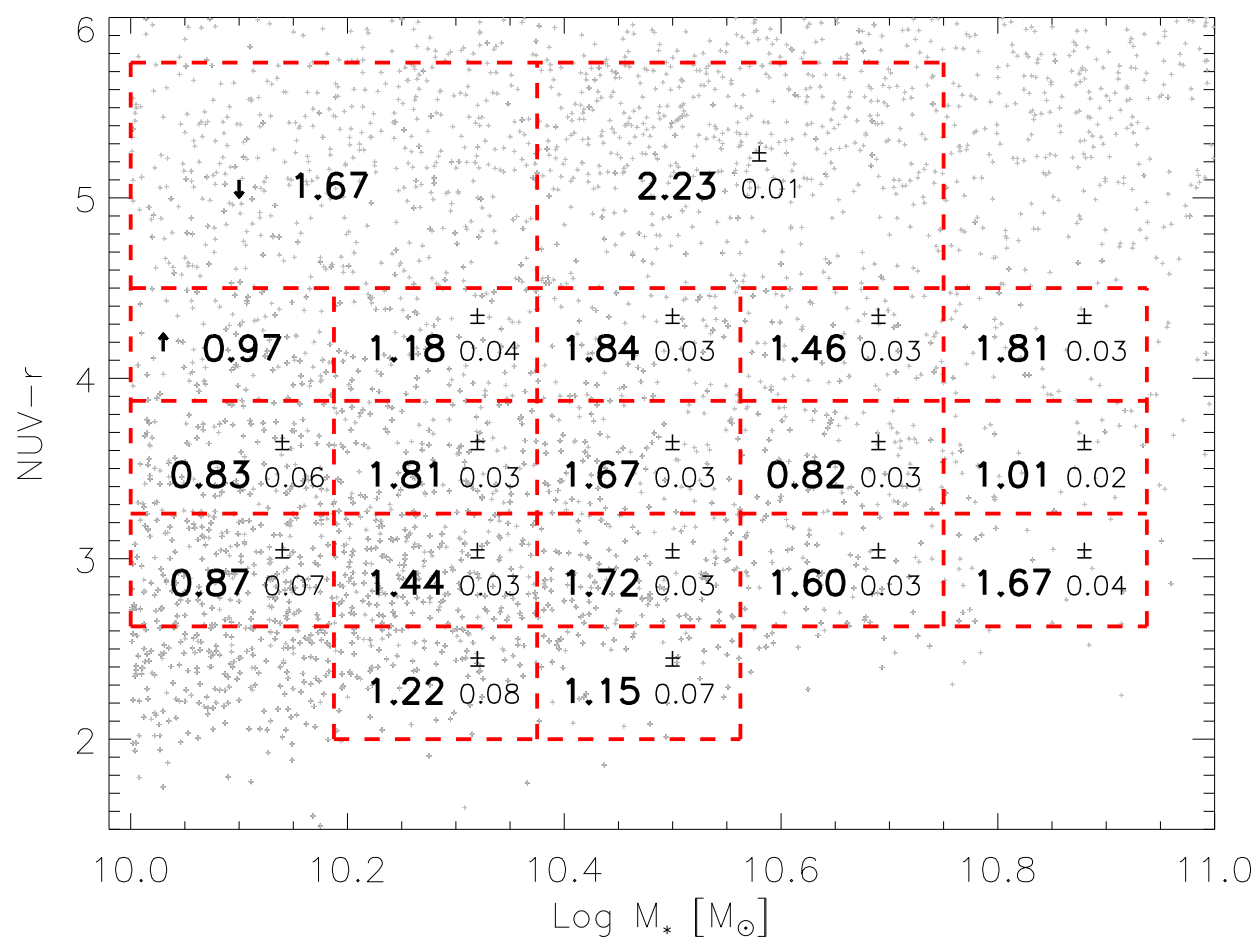

Figure 12. Morphological quenching test. For each bin of NUV $-r$ and $M_{\star}$ the ratio between the gas fractions of the disk-dominated galaxies with $C<2.6 \mathrm{f}(\mathrm{HI})_{D D}$ and the bulge-dominated galaxies with $C>2.6 \mathrm{f}(\mathrm{HI})_{B D}$ is reported. The panel shows the adopted binning with the values measured for each bin. The arrows indicate upper/lower limit if one of the stacked spectra yielded a non-detection. Gray dots show how the targets (LTGs+ETGs) distribute along the plane.

mation of the galactic disk is decoupled from the formation of the bulge. One way that condition (2) could be satisfied is if the disk is formed from gas that accretes well after the bulge formation event, so that the amount and the configuration of the accreted material is not in any way related to properties of the bulge, such as its mass or its velocity dispersion.

We now attempt to establish whether this hypothesis is correct. At fixed total stellar mass, we found that galaxies with larger $C$ have lower gas fractions than galaxies with smaller $C$ (Figure 9, upper panel). If the gas is associated mainly with the disk, then the ratio $\mathrm{M}_{\mathrm{HI}} / M_{* ; \text { disk }}$ should not depend on $C$. We have fit the Weinmann et al. (2009) relation between $\mathrm{B} / \mathrm{T}$ and $C$. Our bi-linear fit result is $C=1.19+2.28 \cdot B / T$. For each galaxy in our sample, we compute $M_{* ; \text { disk }}$. In Figure 13 , we plot $\mathrm{M}_{\mathrm{HI}} / M_{* ; \text { disk }}$ as a function of $\log M_{*}$ for ETG and for sample $A$ galaxies. As can be seen, the difference in gas fraction between the two samples is greatly reduced when the HI mass is divided by the disk stellar mass. For reference, the red and blue lines on the plot show $\mathrm{M}_{\mathrm{HI}} / M_{* ; \text { total }}$, as in Figures 7 and 9 (upper panels).

We note that our result that the Hi fraction of earlytype galaxies depends on the size of the galaxy, but not on its bulge properties, is opposite to what is found for bulge stellar populations. In a recent paper, Graves et al. (2009b) carried out an analysis of 16,000 nearby quiescent galaxies with 3-arcsecond aperture spectra from the Sloan Digital Sky Survey. Their paper demonstrates convincingly that mean stellar age, $[\mathrm{Fe} / \mathrm{H}],[\mathrm{Mg} / \mathrm{H}]$, and $[\mathrm{Mg} / \mathrm{Fe}]$ scale strongly with the velocity dispersion of the bulge, but there is no dependence on $R_{e}$ at a fixed value of $\sigma$. We thus infer that the star formation history of the central bulge does not depend on the size of the galaxy. Our own results show that the present-day gas content of early-types does depend on size. This again argues for bulge and disk formation processes that are decoupled.

2. Galaxies with significant bulge component are not less efficient in turning their available gas into stars. One possible explanation of this result is that the rate of gas consumption in galactic disks is primarily regulated by externally-driven rather than internally-generated instabilities. In recent work, Chakrabarti \& Blitz (2009) present an analysis of the observed perturbations of the Hi disk of the Milky Way and infer the existence of a dark sub-halo that tidally interacted with the Milky Way disk. In addition to dark sub-halos, luminous satellite galaxies are observed to interact with galactic disks. Finally, the dark matter environment at the centers of present day halos is neither static nor in equilibrium. Gao \& White (2006) used the Millennium Simulation to study asymmetries in dark matter halo cores. They found that 20 per cent of cluster haloes have density center separated from barycenter by more than 20 percent of the virial radius, while only 7 percent of Milky Way haloes have such large asymmetries. Because early-type galaxies reside in more massive dark matter halos than latetype galaxies (Mandelbaum et al. 2006), their disks may be subject to considerably larger externally-driven perturbations. All these effects may counteract the stabilizing effect of the bulge. 


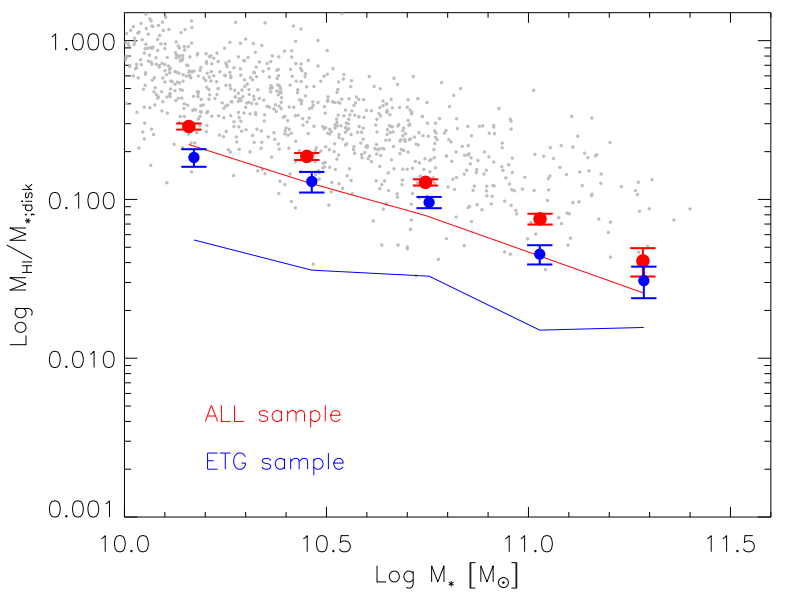

Figure 13. Average disk gas fractions of sample A (red circles) and ETG sample galaxies (blue circles) as a function of total stellar mass $M_{*}$. The lines show the results obtained measuring the total gas fraction, as in Figures 7 and 9 (upper panels). Gray dots show galaxies with ALFALFA detections from sample A.

Further tests of the proposed external origin of the gas in early-type galaxies will come from more detailed analysis of its spatial distribution and kinematics. By studying the relation between gas and stellar angular momentum in earlytype galaxies, one can hope to gain further understanding of how the gas was accreted. Studies of this nature are planned as part of the next generation of integral-field spectroscopy studies of nearby early-type galaxies, e.g. the ATLAS3D survey (http://www-astro.physics.ox.ac.uk/atlas3d/).

\section{APPENDIX A: COMPARISON OF TWO DIFFERENT STACKING PROCEDURES}

In this section we compare two different methods for deriving mean Hi gas fractions from stacked spectra.

1) Stacking Hi fluxes. In the first method, we derive the average $\mathrm{HI}$ gas fraction using the equation

$$
\left\langle\frac{M_{H I}}{M_{*}}\right\rangle=\frac{2.356 \times 10^{5}}{1+\langle z\rangle} \frac{D_{L}^{2}(\langle z\rangle)}{\left\langle M_{*}\right\rangle} \frac{\sum_{i=0}^{N} S_{i} \cdot w_{i}}{\sum_{i=0}^{N} w_{i}},
$$

where $\langle z\rangle$ is the mean redshift of the stacked galaxies and $\left\langle M_{*}\right\rangle$ is their mean stellar mass. The main limitation is that this method may produce biased results if galaxies in the bin span a significant range in redshift or stellar mass. By stacking mainly non-detected spectra, we do not know how much each galaxy contributes to the total signal. This approach will work best if the bins are small. We note that our redshift range is compact enough so that:

$$
D_{L}^{2}(\langle z\rangle) \simeq\left\langle D_{L}(z)\right\rangle^{2} \simeq\left\langle D_{L}^{2}(z) .\right\rangle
$$

We choose to split each stellar mass bin into three sub-bins in redshift: [0.025;0.033],[0.033;0.042],[0.417;0.050]. For each of these sub-bins, we evaluate the mean stellar mass $\left\langle M_{*}\right\rangle$, and split the sample into two further bins according to: $\mathrm{M}_{\star} \gtrless\left\langle M_{*}\right\rangle$. We stack the spectra in each of the 6 sub-bins, measure a flux and evaluate a gas fraction using both the average and median values of $z$ and $M_{*}$. Then we evaluate the final gas fraction by averaging the six values obtained (weighted by the number of objects co-added).

2) Stacking "gas fractions". This approach is described in section 3.2 and is the one that is adopted throughout the main body of this paper.

First, we checked that the rms of the co-added spectra decreases as $1 / \sqrt{N}$; when method (2) is applied, i.e. by multiplying the flux by distance and stellar mass, we are in fact rescaling the noise in the spectra in a sensible way. Results are shown in Figure 14 (panel a): red circles are obtained by stacking "gas fractions", black circles are obtained by stacking fluxes. The black points have been multiplied by the average squared distance and divided by the average $M_{\star}$ in order to have the same units as the red points $\left[\mathrm{mJy} \mathrm{Mpc}{ }^{2} \mathrm{M}_{\odot}^{-1}\right]$. The dashed line is the expected $1 / \sqrt{N}$ dependence, which is still recovered using method (2).

We then compared the results of stacking methods (1) and (2). We divided all the galaxies in sample $A$ that were detected by ALFALFA (detection codes 1+2) into five stellar mass bins. We compare results from the different stacking methods with the results obtained by averaging together the individual catalogued measurements. The results are shown in Figure 14 (panel b), where red circles show results obtained using method (2), and green triangles/blue squares are from method (1), when mean or median values of $z$ and $M_{*}$ are used in equation (A1), respectively. The stars show the results from averaging together the individual detections. Gray dots show the ALFALFA detections. The signal recovered from the stacking is consistent with the mean value of the individual detections for each bin. The two different stacking methods yield results that are also consistent with each other. Small differences of around $\sim 10 \%$ in $\mathrm{M}_{\mathrm{HI}} / M_{*}$ do occur in the two largest mass bins that contain the fewest objects.

We also compared results for the different methods including the non-detections. In Figure 15 we show the same correlations studied in section 4 for sample $A$, computed using different methods. Symbols are the same as described above. Once again, we obtain good consistency.

\section{ACKNOWLEDGMENTS}

SF wishes to thank Genevieve J. Graves and Greg Hallenbeck for useful discussions. We thank the many members of the ALFALFA team who have contributed to the acquisition and processing of the ALFALFA dataset over the last six years.

RG and MPH are supported by NSF grant AST0607007 and by a grant from the Brinson Foundation.

The Arecibo Observatory is part of the National Astronomy and Ionosphere Center, which is operated by Cornell University under a cooperative agreement with the National Science Foundation.

GALEX is a NASA Small Explorer, launched in 2003 April. We gratefully acknowledge NASA's support for construction, operation and science analysis for the GALEX mission, developed in cooperation with the Centre National 

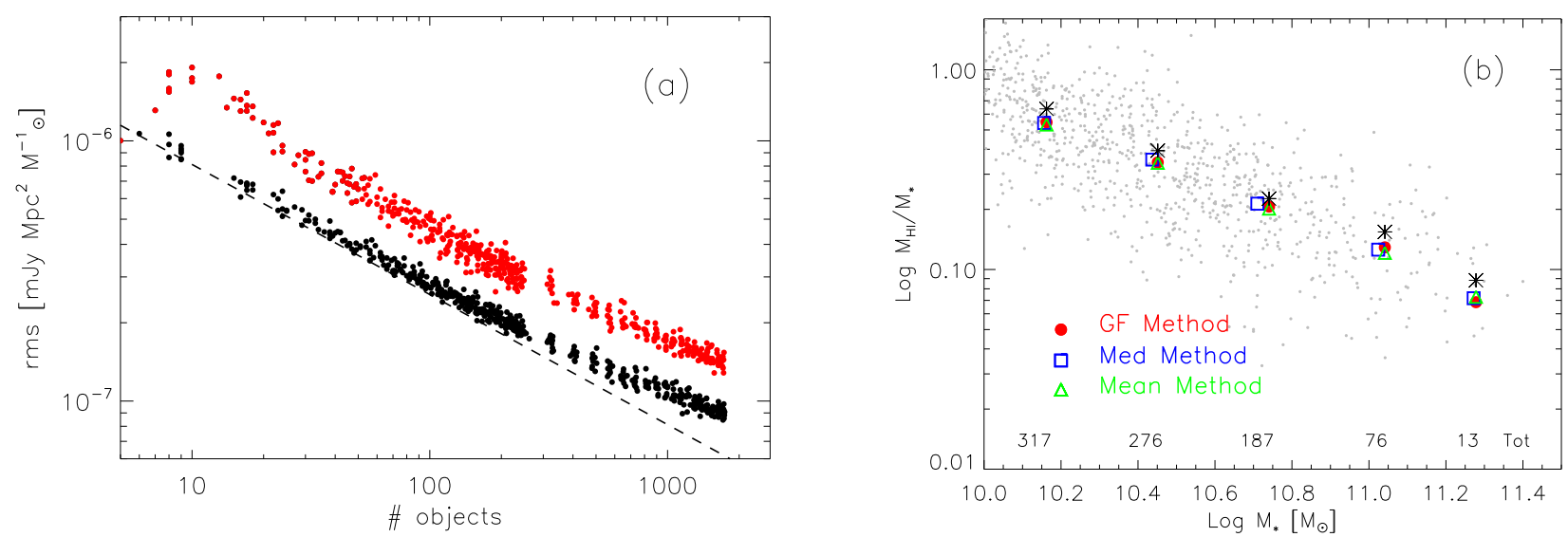

Figure 14. (a) Dependence of the rms of the stacked spectra on the number of objects stacked. The red dots are obtained by stacking "gas fractions". The black ones by stacking HI line fluxes. The dashed line is the expected $1 / \sqrt{N}$ dependence (note that the black points have been multiplied by the average squared distance and divided by the average $M_{\star}$ in order to have the same units as the red points $\left.\left[\mathrm{mJy} \mathrm{Mpc}^{2} \mathrm{M}_{\odot}^{-1}\right]\right)$. (b) Comparison of average gas fractions obtained with the two different stacking methods applied to sample A galaxies with ALFALFA detections. Red circles are averages obtained using method 2, and green triangles/blue squares are from method (1), when mean or median values of $z$ and $M_{*}$ are used in equation (A1), respectively. The stars are the means obtained from averaging the catalogued ALFALFA fluxes. Gray dots show the ALFALFA detections. The numbers of objects co-added in each bin is reported below.
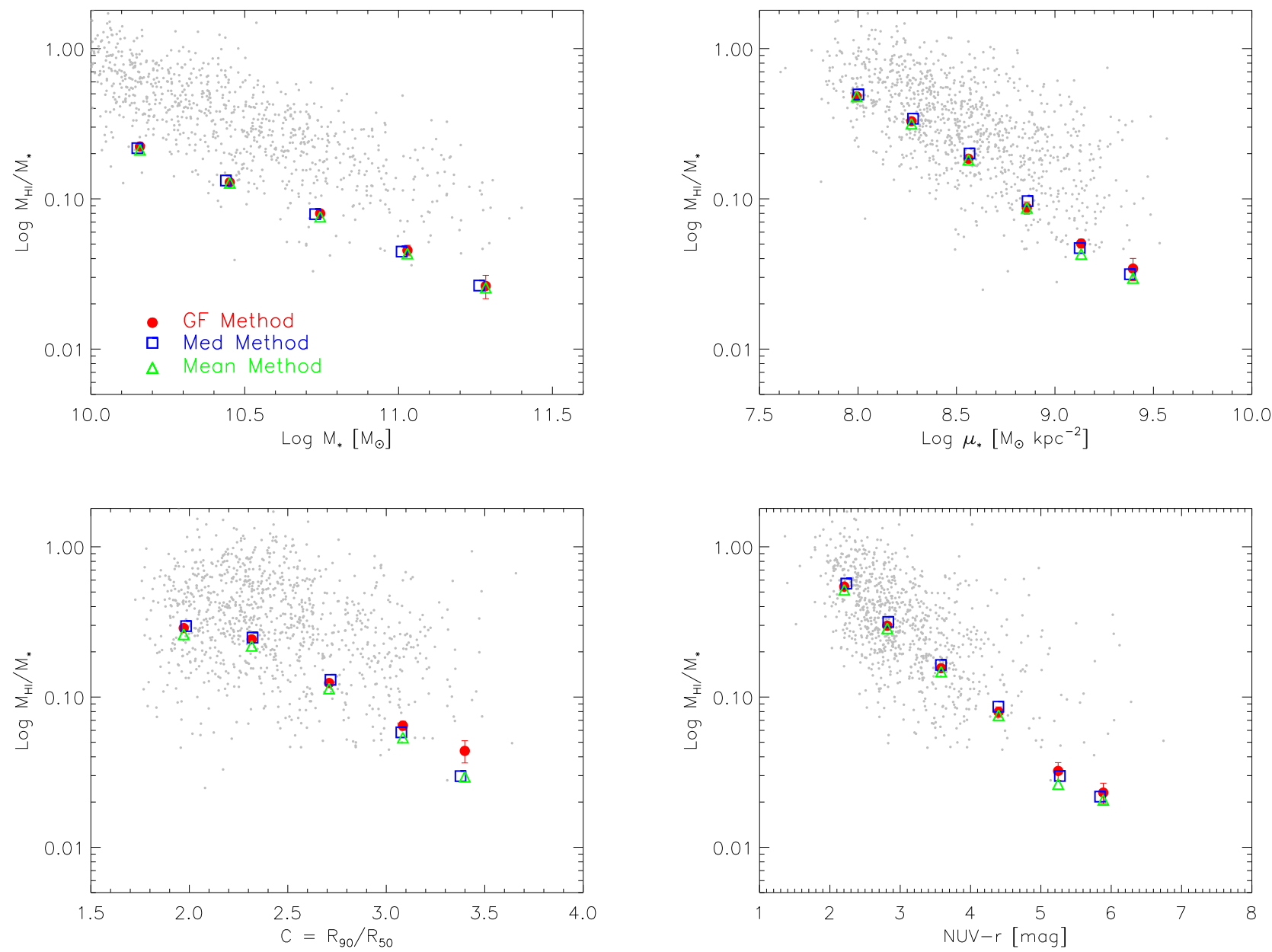

Figure 15. Comparison of average gas fractions obtained with the two different stacking methods, applied to all sample $A$ galaxies. Symbols and colours are the same as Figure 14 
d'Etudes Spatiales (CNES) of France and the Korean Ministry of Science and Technology.

Funding for the SDSS and SDSS-II has been provided by the Alfred P. Sloan Foundation, the Participating Institutions, the National Science Foundation, the U.S. Department of Energy, the National Aeronautics and Space Administration, the Japanese Monbukagakusho, the Max Planck Society and the Higher Education Funding Council for England. The SDSS web site is http://www.sdss.org/.

The SDSS is managed by the Astrophysical Research Consortium for the Participating Institutions. The Participating Institutions are the American Museum of Natural History, Astrophysical Institute Potsdam, University of Basel, University of Cambridge, Case Western Reserve University, University of Chicago, Drexel University, Fermilab, the Institute for Advanced Study, the Japan Participation Group, Johns Hopkins University, the Joint Institute for Nuclear Astrophysics, the Kavli Institute for Particle Astrophysics and Cosmology, the Korean Scientist Group, the Chinese Academy of Sciences (LAMOST), Los Alamos National Laboratory, the Max-Planck-Institute for Astronomy (MPIA), the Max-Planck-Institute for Astrophysics (MPA), New Mexico State University, Ohio State University, University of Pittsburgh, University of Portsmouth, Princeton University, the United States Naval Observatory and the University of Washington.

\section{REFERENCES}

Adelman-McCarthy J. K., et al., 2008, ApJS, 175, 297

Baldry, I. K., Glazebrook, K., Brinkmann, J. et al. 2004, ApJ, 600, 681

Baldry I. K., Balogh M. L., Bower R. G., Glazebrook K., Nichol R. C., Bamford S. P., Budavari T., 2006, MNRAS, 373, 469

Bernardi, M., et al. 2003, ApJ, 125, 1817

Bregman, J. N., Hogg, D. E., \& Roberts, M. S. 1992, ApJ, 387,484

Brinchmann J., Charlot S., White S. D. M., Tremonti C., Kauffmann G., Heckman T., Brinkmann J., 2004, MNRAS, 351, 1151

Carilli C. L., et al., 2008, ApJ, 689, 883

Catinella, B., Haynes, M. P., Giovanelli, R., Gardner, J. P., \& Connolly, A. J. 2008, ApJL, 685, L13

Catinella B., et al., 2010, MNRAS, 403, 683 (GASS-1)

Chakrabarti S., Blitz L., 2009, MNRAS, 399, L118

Chengalur, J. N., Braun, R., \& Wieringa, M. 2001, A\&A, 372,768

di Serego Alighieri, S., et al. 2007, A\&A, 474, 851

Efron, B. 1982, CBMS-NSF Regional Conference Series in Applied Mathematics, Philadelphia: Society for Industrial and Applied Mathematics (SIAM), 1982

Gadotti, D. A. 2009, MNRAS, 393, 1531

Gao L., White S. D. M., 2006, MNRAS, 373, 65

Giovanelli, R., et al. 1997, AJ, 113, 22

Giovanelli, R., et al. 1997, AJ, 113, 53

Giovanelli, R. et al. 2005, AJ, 130, 2598

Giovanelli, R. et al. 2007, AJ, 133, 2569

Graves, G. J., Faber, S. M., \& Schiavon, R. P. 2009a, ApJ, 693,486
Graves, G. J., Faber, S. M., \& Schiavon, R. P. 2009b, ApJ, 698, 1590

Grossi, M., et al. 2009, A\&A, 498, 407

Haynes, M. P., \& Giovanelli, R. 1984, AJ, 89, 758

Helmboldt, J. F. 2007, MNRAS, 379, 1227

Hodge J. A., Zeimann G. R., Becker R. H., White R. L., 2009, AJ, 138, 900

Johnson, B. D., et al. 2007, ApJS, 173, 377

Kauffmann, G., et al. 2003, MNRAS, 341, 33

Kauffmann, G., et al. 2003, MNRAS, 341, 54

Kent, B. R. et al. 2008, AJ, 136, 713

Knapp, G. R., Turner, E. L., \& Cunniffe, P. E. 1985, AJ, 90, 454

Lah, P., et al. 2007, MNRAS, 376, 1357

Lah, P., et al. 2009, MNRAS, 399, 1447

Mandelbaum R., Seljak U., Kauffmann G., Hirata C. M., Brinkmann J., 2006, MNRAS, 368, 715

Martig, M., Bournaud, F., Teyssier, R., \& Dekel, A. 2009, ApJ, 707, 250

Martin, D. C. et al. 2005, ApJ, 619, L1

Morganti, R., et al. 2006, MNRAS, 371, 157

Ostriker J. P., Peebles P. J. E., 1973, ApJ, 186, 467

Roberts, M. S. 1963, ARAA, 1, 149

Roberts, M. S., \& Haynes, M. P. 1994, ARAA, 32, 115

Saintonge, A. 2007, AJ, 133, 2087

Salim, S. et al. 2007, ApJS, 173, 267

Schawinski, K. et al. 2007, MNRAS, 382, 1415

Schiminovich, D., et al. 2010, MNRAS, 1288, in press

Shen S. et al. 2006, MNRAS, 369, 1639

Shostak, G. S., \& Allen, R. J. 1980,A\&A, 81, 167

Stierwalt, S., et al. 2009, AJ, 138, 338

Tremonti C. A., et al., 2004, ApJ, 613, 898

Tukey, J. W. 1977, Addison-Wesley Series in Behavioral Science: Quantitative Methods, Reading, Mass.: AddisonWesley, 1977

Verheijen, M., et al. 2007, ApJ, 668, L9

Wang, J., Overzier, R., Kauffmann, G., von der Linden, A., \& Kong, X. 2010, MNRAS, 401, 433

Wardle, M., \& Knapp, G. R. 1986, AJ, 91, 23

Weinmann, S. M., et al. 2009, MNRAS, 394, 1213

York, D. G., et al. 2000, AJ, 120, 1579

Zibetti S., White S. D. M., Schneider D. P., Brinkmann J., 2005, MNRAS, 358, 949 


\begin{tabular}{|c|c|c|c|c|c|c|c|c|c|c|}
\hline$x$ & $\langle x\rangle$ & $a$ & $\begin{array}{c}\left\langle\mathrm{M}_{\mathrm{HI}} / \mathrm{M}_{\star}\right\rangle \\
\text { Sample A }\end{array}$ & $\mathrm{N}$ & $\begin{array}{c}\left\langle\mathrm{M}_{\mathrm{HI}} / \mathrm{M}_{\star}\right\rangle \\
\text { ETG sample }\end{array}$ & $\mathrm{N}$ & $\begin{array}{c}\left\langle\mathrm{M}_{\mathrm{HI}} / \mathrm{M}_{\star}\right\rangle \\
\text { ETG sample } 1\end{array}$ & $\mathrm{~N}$ & $\begin{array}{c}\left\langle\mathrm{M}_{\mathrm{HI}} / \mathrm{M}_{\star}\right\rangle \\
\text { ETG sample 2 }\end{array}$ & $\mathrm{N}$ \\
\hline \multirow[t]{5}{*}{$\log \mathrm{M}_{\star}$} & 10.16 & $-0.78 \pm 0.03$ & $0.222 \pm 0.008$ & 1734 & $0.056 \pm 0.009$ & 493 & $0.091 \pm 0.011$ & 311 & $0.118 \pm 0.019$ & 139 \\
\hline & 10.45 & & $0.128 \pm 0.005$ & 1538 & $0.036 \pm 0.005$ & 566 & $0.056 \pm 0.006$ & 389 & $0.049 \pm 0.006$ & 294 \\
\hline & 10.74 & & $0.079 \pm 0.004$ & 1025 & $0.033 \pm 0.004$ & 516 & $0.043 \pm 0.005$ & 351 & $0.041 \pm 0.005$ & 328 \\
\hline & 11.03 & & $0.044 \pm 0.004$ & 430 & $0.015 \pm 0.004$ & 246 & $0.018 \pm 0.003$ & 183 & $0.014 \pm 0.004$ & 178 \\
\hline & 11.28 & & $0.026 \pm 0.005$ & 63 & $0.016 \pm 0.004$ & 43 & $0.007^{*}$ & 32 & $0.009^{*}$ & 25 \\
\hline \multirow{5}{*}{$\begin{array}{c}C \\
\left(R_{90} / R_{50}\right)\end{array}$} & 1.97 & $-0.59 \pm 0.03$ & $0.288 \pm 0.013$ & 420 & - & - & - & - & - & - \\
\hline & 2.32 & & $0.242 \pm 0.008$ & 1149 & - & - & - & - & - & - \\
\hline & 2.71 & & $0.124 \pm 0.006$ & 1464 & - & - & - & - & - & - \\
\hline & 3.08 & & $0.064 \pm 0.004$ & 1409 & - & - & - & - & - & - \\
\hline & 3.40 & & $0.044 \pm 0.007$ & 287 & - & - & - & - & - & - \\
\hline \multirow[t]{6}{*}{$\log \mu_{\star}$} & 7.99 & $-0.87 \pm 0.03$ & $0.481 \pm 0.025$ & 193 & - & - & - & - & - & - \\
\hline & 8.27 & & $0.328 \pm 0.011$ & 625 & $0.293 \pm 0.082$ & 8 & $0.336 \pm 0.109$ & 5 & $0.435 \pm 0.154$ & 3 \\
\hline & 8.56 & & $0.185 \pm 0.008$ & 1003 & $0.112 \pm 0.018$ & 148 & $0.107 \pm 0.019$ & 120 & $0.139 \pm 0.047$ & 41 \\
\hline & 8.86 & & $0.087 \pm 0.008$ & 1371 & $0.077 \pm 0.006$ & 661 & $0.071 \pm 0.007$ & 461 & $0.073 \pm 0.010$ & 258 \\
\hline & 9.13 & & $0.050 \pm 0.003$ & 1237 & $0.043 \pm 0.004$ & 857 & $0.037 \pm 0.004$ & 591 & $0.036 \pm 0.004$ & 535 \\
\hline & 9.40 & & $0.034 \pm 0.006$ & 287 & $0.035 \pm 0.008$ & 183 & $0.029 \pm 0.011$ & 89 & $0.020 \pm 0.005$ & 124 \\
\hline \multirow[t]{6}{*}{$\mathrm{NUV}-r$} & 2.20 & $-0.38 \pm 0.01$ & $0.543 \pm 0.024$ & 209 & $0.351 \pm 0.091$ & 14 & $0.301 \pm 0.121$ & 10 & $0.569 \pm 0.293$ & 3 \\
\hline & 2.82 & & $0.298 \pm 0.009$ & 855 & $0.253 \pm 0.019$ & 123 & $0.220 \pm 0.020$ & 77 & $0.247 \pm 0.038$ & 29 \\
\hline & 3.58 & & $0.156 \pm 0.007$ & 760 & $0.127 \pm 0.011$ & 211 & $0.125 \pm 0.014$ & 127 & $0.152 \pm 0.020$ & 74 \\
\hline & 4.40 & & $0.079 \pm 0.006$ & 609 & $0.073 \pm 0.009$ & 278 & $0.072 \pm 0.013$ & 180 & $0.084 \pm 0.011$ & 118 \\
\hline & 5.24 & & $0.032 \pm 0.004$ & 909 & $0.032 \pm 0.005$ & 669 & $0.027 \pm 0.005$ & 468 & $0.022 \pm 0.004$ & 387 \\
\hline & 5.89 & & $0.023 \pm 0.004$ & 621 & $0.024 \pm 0.004$ & 490 & $0.014 \pm 0.004$ & 349 & $0.021 \pm 0.004$ & 315 \\
\hline \multirow[t]{6}{*}{$\log \sigma$} & 1.90 & - & $0.149 \pm 0.014$ & 287 & $0.077 \pm 0.020$ & 76 & $0.085 \pm 0.026$ & 50 & $0.058 \pm 0.016$ & 99 \\
\hline & 2.01 & & $0.135 \pm 0.009$ & 483 & $0.089 \pm 0.014$ & 169 & $0.074 \pm 0.016$ & 114 & $0.059 \pm 0.006$ & 302 \\
\hline & 2.10 & & $0.077 \pm 0.005$ & 738 & $0.054 \pm 0.007$ & 353 & $0.047 \pm 0.008$ & 234 & $0.059 \pm 0.010$ & 136 \\
\hline & 2.20 & & $0.053 \pm 0.005$ & 711 & $0.041 \pm 0.004$ & 439 & $0.042 \pm 0.006$ & 287 & $0.048 \pm 0.006$ & 252 \\
\hline & 2.29 & & $0.023 \pm 0.003$ & 436 & $0.022 \pm 0.003$ & 322 & $0.022 \pm 0.004$ & 222 & $0.025 \pm 0.004$ & 246 \\
\hline & 2.40 & & $0.010 \pm 0.002$ & 167 & $0.012 \pm 0.002$ & 141 & $0.008^{*}$ & 112 & $0.009^{*}$ & 123 \\
\hline
\end{tabular}

Table A1. Average gas fractions for the samples shown in Figures 7.10 sample A (Fig. 7 and 8 red circles), the main ETG sample (Fig. 9 and 10 blue circles), and the two ETG sub-samples defined with more stringent cuts on $C$ and inclination (Fig. 9 and 10 black and cyan circles). For sample $A$ we also report the slopes $a$ (third column) of the relations $\log \left\langle\mathrm{M}_{\mathrm{HI}} / \mathrm{M}_{\star}\right\rangle=a\langle x\rangle+b$, where $x$ is the quantity listed in the 1 st column. 